\title{
Intrinsic synergistic-topological mechanism versus synergistic-topological matrix in microtubule self-organization
}

\author{
Vlado A Buljan ${ }^{1,2,3^{*}}$, R M Damian Holsinger ${ }^{1,4}$, Brett D Hambly ${ }^{5}$, Vangelis Kanellis ${ }^{1}$, Elie Matar ${ }^{1}$, Xanthe Larkin $^{1}$,
} Guo Jun Liü, John J Bohorquez-Florez and Richard B Banati ${ }^{1}, 3,6$

\author{
* Correspondence: \\ vlado.buljan@sydney.edu.au \\ ${ }^{1}$ Brain and Mind Research Institute, \\ Sydney Medical School, The \\ University of Sydney, Sydney, NSW \\ 2050, Australia \\ 2Department of Physiology, Sydney \\ Medical School, The University of \\ Sydney, Sydney, NSW 2006, \\ Australia \\ Full list of author information is \\ available at the end of the article
}

\begin{abstract}
Background: In this body of work we investigate the synergistic-topological relationship during self-organization of the microtubule fiber in vitro, which is composed of straight, axially shifted and non-shifted, acentrosomal microtubules under crowded conditions.

Methods: We used electron microscopy to observe morphological details of ordered straight microtubules. This included the observation of the differences in length distribution between microtubules in ordered and non-ordered phases followed by the observation of the formation of interface gaps between axially shifted and ordered microtubules. We performed calculations to confirm that the principle of summation of pairwise electrostatic forces act between neighboring microtubules all their entire length.

Results: We have shown that the self-organization of a microtubule fiber imposes a variety of topological restrictions onto its constituting components: (a) tips of axially shifted neighboring microtubules are not in direct contact but rather create an interface gap; (b) fibers are always composed of a restricted number of microtubules at given solution conditions; (c) the average length of microtubules that constitute a fiber is always shorter than that of microtubules outside a fiber; (d) the length distribution of microtubules that constitute a fiber is narrower than that of microtubules outside a fiber and this effect is more pronounced at higher GTP-tubulin concentrations; (e) a cooperative motion of fiber microtubules due to actualization of the summation principle of pairwise electrostatic forces; (f) appearance of local GTP-tubulin depletion immediately in front of the tips of fiber microtubules.

Conclusion: Overall our data indicate that under crowded conditions in vitro, the self-organization of a microtubule fiber is governed by an intrinsic synergistic-topological mechanism, which in conjunction with the topological changes, GTP-tubulin depletion, and cooperative motion of fiber constituting microtubules, may generate and maintain a synergistic-topological matrix. Failure of the mechanism to form biologically feasible microtubule synergistic-topological matrix may, per se, precondition tumorigenesis.
\end{abstract}

\section{Springer}

\section{Background}

Microtubules are functionally and critically involved in the major events of intracellular organization including cell morphology [1], cellular motility [2], cell division [3], intracellular transport [4], signal transduction [5] and memory encoding [6].

2014 Buljan et al.; licensee Springer on behalf of EPJ. This is an Open Access article distributed under the terms of the Creative Commons Attribution License (http://creativecommons.org/licenses/by/4.0), which permits unrestricted use, distribution, and reproduction in any medium, provided the original work is properly credited. 
In order to perform their roles accurately and efficiently, it is essential for microtubules to undergo highly coordinated behaviour, which implicates diverse forms of crosstalk phenomena including crosstalk between microtubules and other cytoskeletal components, between two or more individual microtubules or between different domains of single microtubules [3,7-15]. It has been recognized that crass-talk between cytoskeletal components (including microtubules) is conditio sine qua non for synergy to occur in the system [16]. Therefore, it is not surprising that in the presence of highly correlated motion of microtubules, combined with crosstalk, the presence of different modes of synergistic mechanism may be observed [17-19].

It has been shown that the mitotic spindle is a self-made machine, i.e., it can self-organize under biologically poor conditions (in the absence of centrosome and chromatin) [20-22]. To explain these findings it has been proposed that the physical state of the cytoplasm surrounding chromosomes is changed in a way that favors microtubule growth and self-organization into the spindle [21]. Indeed, it has recently been shown that self-organization of anastral spindles are guided by the synergy of dynamic instability, autocatalytic microtubule production and spatial signaling gradients [19]. Taken together these works may support the idea that some synergy operating mode(s) may be inherent to microtubule systems and local solution conditions.

The idea that the physical conditions of the cytoplasm may favor microtubule mitotic spindle self-formation is similar to the concept of a spindle matrix. The concept of the spindle matrix was introduced in order to explain the mechanics of complex movement of microtubules in mitotic spindles and its highly correlated behavior in relation to chromosome movement during cellular division [23]. Recent elaboration of the matrix concept in the study of spatio-temporal control of complex reorganization of microtubules in mitotic spindles during mitosis have shown that the concept requires further development to include molecular details, pliable structural dynamic features and cross-linking features $[17,24,25]$. As noted above, cross-linking may endow the system with cross-talk ability, which is indeed a synergistic feature. Therefore, it follows that the concept of the spindle matrix should also be bestowed with appropriate synergistic aspects. It has been suggested that the matrix exhibits elastic gel properties [26]. On the other hand, the high resistivity of the self-organized pattern in microtubules (in vitro) to disruptive forces may suggest that a similar matrix is formed, which, in association with the spatio-temporal pattern of microtubules in vitro, exhibits properties of an elastic gel [27]. Therefore, it would be beneficial to extend the study of the microtubule spindle matrix to the analogous matrix of any self-organized spatio-temporal microtubule pattern, especially those that include a far simpler pattern that is formed under solution conditions in vitro.

To investigate the possibility that intrinsic synergistic mechanisms in conjunction with the topology of spatio-temporally self-organized microtubules may form, we define this as microtubule synergistic-topological matrix. Under biologically reduced solution conditions in vitro, we have used a simple system comprising GTP-tubulin and a small pool of classical microtubule associated proteins (see Methods).

Under the crowded conditions, in terms of GTP-tubulin concentration, we have observed self-organized spatio-temporal patterns of microtubules (fibers). The crowded conditions, if they are strong enough, may reduce the degree of freedom of motion of protein molecule resulting in anisotropic motion [28]. 
In order to solve the problem of blind mason, during its addition to the tip, GTP-tubulin has to have significant degree of freedom of motion (rotational and translational diffusion) [29]. Therefore, by limiting the accommodating space for free rotation and translation of GTP-tubulin molecule, the highly crowded conditions are in conflict with solving the problem of blind mason during microtubule assembly. However, we have shown that crowded conditions in our experiments were not too strong so that the problem of blind mason is properly solved.

The fibers have always imposed some topological restrictions onto the constituent microtubules and have self-generated local depletion of GTP-tubulin in the immediate vicinity of the tips of microtubules. Obviously, affecting the shape of the fiber by acting at the microtubule tips, the local depletion of GTP-tubulin determines the dynamic of microtubule fiber in axial direction. In addition, there is strong mutual interdependence between the topology of microtubules and local solution conditions. Therefore, the spatio-temporal pattern of microtubules could be treated as an integrated composite of microtubule patterns itself and locally associated specific solution conditions. In an analogy to the spindle matrix, the nonlinear physics of integrated composites could be mathematically modeled by means of some sort of matrix.

An important implication of our data is that the self-organized microtubules are co-localized, interconnected and interdependent. These three features are critical conditions for crosstalk and consequently for intrinsic synergistic mechanisms to operate in the system as well [16]. The physical basis of these features is the principle of summation of electrostatic pairwise forces, which acts between neighboring microtubules all the way along their lengths [30]. Our calculations show that under the crowded conditions of our experiments the pairwise electrostatic forces follow the principle of summation. Therefore, the shape and the dynamic of fiber along its perpendicular direction are determined by the action of the principle of the forces summation across the constitutional microtubules. In addition, the principle endows constitutional microtubules with cooperative behavior.

Taken together the depletion of GTP-tubulin and the action principle of pairwise forces summation are critical and independent ingredients in the synergistic mechanism and the formation of synergistic-topological matrix .

As for the rationale of our data we propose that, under crowded conditions in vitro, the self-organization of microtubule fibers is governed by an intrinsic synergistic mechanism that in conjunction with the topological changes in microtubules may generate a synergistic-topological matrix.

Synergistic-topological mechanisms are pivotal in the formation and maintenance of a matrix. Failure of intrinsic synergistic-topological mechanisms to form and maintain biologically feasible microtubule synergistic-topological matrices may precondition the system to decline into biologically non-feasible events such as aneuploidy during mitosis that may in turn precondition the system for tumorigenesis $[15,31,32]$.

\section{Methods}

\section{The systems tested}

Unless stated otherwise all experiments were performed with microtubules in MES buffer containing (in mM): 100 MES, 1 EGTA, $0.5 \mathrm{MgCl}_{2}$, 1.0 GTP, pH 6.6. Microtubules were 
reconstituted at different concentrations of microtubule protein (MTP). MTP refers to 95\% tubulin $+5 \%$ MAPs [33].

Microtubule assembly was initiated by the addition of $1 \mathrm{mM} \mathrm{GTP}$ at 36?C. A stationary state of assembly was reached in 30 minutes. Microtubule protein was prepared using bovine brain as described elsewhere [33].

In order to observe the effect of calcium on microtubule morphology and their ordering, in some experiments we added an excess of $1 \mathrm{mM}$ free calcium $\left(\mathrm{Ca}^{2+}\right)$.

\section{Electron microscopy: the morphology of ordered rudimentary microtubule phases on a micrometer scale}

Our goal was to observe morphological details of ordered straight microtubules. This included the observation of the differences in length distribution between microtubules in ordered and non-ordered phases followed by the observation of the formation of interface gaps between axially shifted and ordered microtubules. For this purpose the specimens were viewed using an analytical Philips CM12 transmission electron microscope (TEM) at the Australian Key Centre for Microscopy and Microanalysis, at the University of Sydney. EM images were obtained using a Gatan multiscan CCD camera and an exposure time of $1.51 \mathrm{~s}$.

In order to be viewed by EM, microtubules were prepared according to Langfords method [34]. Briefly, samples of microtubules containing different MTP concentrations were left to assemble for $60 \mathrm{~min}$ at 36? C. Microtubules were stabilised by adding 50\% sucrose, fixed and stained with $2 \%$ uranyl acetate on 200 mesh grids. Samples were left overnight at room temperature prior to microscopic examination.

Microtubule lengths were measured using the Prism Image Analysis System (Analytical Vision Inc, CA). The length of microtubules (both, curved and straight) has been measured as the average contour microtubule length. In further text the average contour microtubule length will be referred to as microtubule length. We were primarily interested in microtubules of straight morphology. For this purpose our buffer did not contain any excess $\mathrm{Ca}^{2+}$. However, for the purpose of comparison we prepared curved microtubules by adding an excess of $1 \mathrm{mM} \mathrm{Ca}^{2+}$ to solutions prior to microtubule assembly [35].

Microtubule samples were applied to coverslips (No 1 Superior Micro Cover Glasses W. Germany) using the procedures described in [36].

\section{Results}

Using transmission electron microscopy, we have studied the basic parameters of selforganized microtubule fibers under crowded conditions in vitro. The basic fiber parameters include: fiber size (the total number of constituent microtubules), the average length and length distribution of microtubules that constitute the fiber. We have also observed numerous, nanometer size, microtubule free space (which we termed interface gap) between the tips of approaching microtubules within fibers. In addition, our calculations have revealed that total depletion of GTP-tubulin can occur in the immediate vicinity of the tips of microtubule fibers, that is more pronounced versus more crowded conditions. 
Symmetry breaking per se indicates the presence of a synergistic mechanism(s)

Formation of self-organized microtubule fibers and their spatial networks are accompanied by symmetry breaking, which per se indicates the presence of a synergistic $\operatorname{mechanism}(\mathrm{s})[37,38]$.

\section{Straight microtubule fibers}

We have observed straight microtubules spontaneously organize into straight fibers at 36 ? $\mathrm{C}$ in the presence of $7 \mathrm{mg} / \mathrm{ml}$ MTP and calcium chelated by $1 \mathrm{mM}$ EGTA (Figure 1A). Strictly speaking the fiber in Figure $1 \mathrm{~A}$ is not constituted purely by the straight microtubules. A very few slightly curved microtubules can be seen as apparently part of the fiber structure. However, bearing in mind that the fibers geometrical, physical and selforganizational features are critically determined by the majority of straight microtubules, we will continue to use term straight microtubule fibers .

Obviously, straight fibers reflect distinct topological relations among the constituent microtubules. Single microtubules were axially shifted relative to their neighbors in the fiber. In this manner, fibers might extend by several average microtubule lengths. Furthermore, multitudes of straight microtubule-based fibers may spontaneously create a network, i.e. they can form a discrete spatial pattern of a higher topological complexity (Figure 1B) at higher MTP concentrations $(10 \mathrm{mg} / \mathrm{ml})$. If microtubules are predominantly straight, then they can maintain a partial parallel ordering pattern at some locations, while they cross each other multiple times elsewhere, forming star-like patterns. Additionally, some locations maybe free of microtubules while they densely populate other locations. Consequently, the initially homogenous space of microtubule multitudes become heterogeneous through symmetry breaking, i.e. differentiated into three regions: (a) microtubule free regions, (b) regions consisting of star-like microtubule patterns and (c) the railroad regions of parallel microtubules. Admittedly, massive spatio-temporal correlations must be involved in this symmetry breaking phenomena that per se provides evidence of the action of intrinsic synergy.
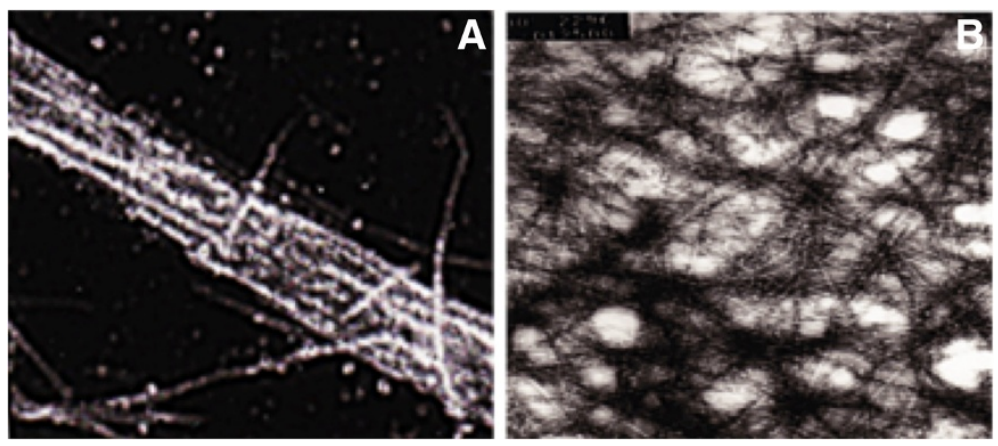

Figure 1 Self-organisation of straight microtubules, at crowded conditions in vitro is guided by breaking the symmetry of initially homogenous space, that reflects the presence of massive spatio-time correlations, i.e. immanent principle of synergy. A. Fibers constituted by straight microtubules. Electron-microscopy (EM), filtered, image at 12500, in vitro at $7 \mathrm{mg} / \mathrm{ml}$ of microtubule protein concentration, and 360C. B. Straight fibers spontaneously self-organize into a spatial patterns of higher complexity (network) in vitro, at higher microtubule protein (MTP) concentration $(10 \mathrm{mg} / \mathrm{ml})$. Predominantly straight microtubules retain partial parallel ordering pattern in some locations, they are multiple crossing each other at other locations, while microtubule free locations are formed as well. 


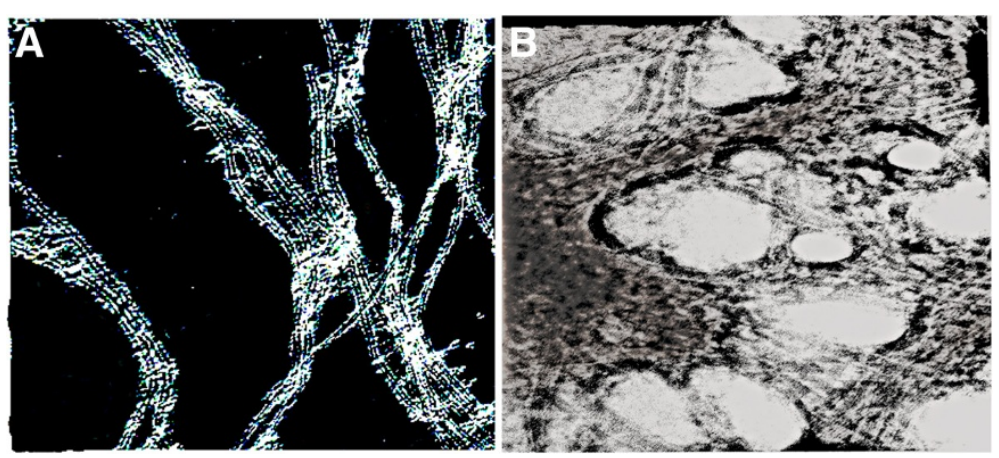

Figure 2 Self-organisation of curved microtubules, at crowded conditions in vitro, is guided by breaking the symmetry of initially homogenous space, that reflects the presence of massive spatio-time correlations, i.e. immanent principle of synergy. Electron-microscopy (EM), filtered image of microtubules fibers at 12500. A. Several curved and entangled fibers constituted by curved microtubules, at $7 \mathrm{mg} / \mathrm{ml}$ MTP and $1 \mathrm{mM} \mathrm{Ca2+}$ in excess. B. Curved fibers spontaneously self-organize into a spatial patterns of higher complexity (network) in vitro, at higher microtubule protein (MTP) concentration $(10 \mathrm{mg} / \mathrm{ml})$, and $1 \mathrm{mM} \mathrm{Ca2+}$ in excess. Curved fibers are constituted of partially parallel curved microtubules, they are multiple crossing each other at other locations, while microtubule free locations are formed as well.

\section{Curved microtubule fibers}

Although curved as a whole, microtubules may retain partial parallel ordering, thus forming partially curved fibers. An example of curved fibers is seen in Figure 2A, at $7 \mathrm{mg} / \mathrm{ml} \mathrm{MTP} \mathrm{in} \mathrm{the} \mathrm{presence} \mathrm{of} \mathrm{excess} \mathrm{calcium.} \mathrm{At} \mathrm{a} \mathrm{higher} \mathrm{MTP} \mathrm{concentration}$ $(10 \mathrm{mg} / \mathrm{ml})$ curved fibers spontaneously create a spatial network (Figure 2B). Here, curved microtubules retain a parallel ordering in some locations, while crossing each other elsewhere. Topological relations are different here in comparison to a fiber formed by straight microtubules. Furthermore, some spatial domains are filled with microtubules while others are microtubule free. Curved microtubules, like straight microtubules, are also increasingly breaking the spatial symmetry of the initially homogenous space, making it increasingly more heterogeneous and therefore organizationally (i.e. synergistically) more complex.

\section{More details about self-organization of straight and curved microtubule forms}

When the curved microtubules are dominant constituent of the curved fiber such as in the case shown in Figure 2A, then their average curvature corresponds to the curvature of the fiber itself within the standard deviation shown below. Using the circle approximation, we found that the average curvature of the curved fiber microtubules in Figure $2 \mathrm{~A}$ is $0.0015 ? 0.00028 \mathrm{~nm}^{-1}$.

This situation is different when the curved microtubules are the minority among the dominant population of the straight microtubules constituting the fiber in Figure 1A. We have estimated that due to presence of impurity, constituted of a few curved microtubules, the width of the whole fiber in Figure 1A is enlarged on average by the factor 1.21. In terms of microtubule diameter and the distance between neighboring microtubules, this enlargement is equivalent to the sum of outer diameter of individual microtubules and the distance between neighboring microtubules. However, this enlargement does not 
appear to affect the fiber straightness. Also, it does not significantly affect the ratio between its length and width. Thus, the fiber still has straight and distinctly extended form.

The other population of rarely found curved and straight microtubules, located randomly across the fiber and eventually near the fiber, we did not consider to be a part of fiber structure. However, we assume them to be a part of non-ordered microtubule population. This population cannot be self-organized because after the fibers formation, the concentration of the rest of microtubules population is decreased below the critical concentration for self-organization.

To illustrate the complexity and the strength of segregation and alignment of straight and curved microtubule forms we use a very simple example of hybrid microtubules (Figure 3). Hybrid microtubule is composed of intrinsically curved and straight parts. Depending on the relative size of its parts the following may happen, straight parts for example are driven into simple alignment, while some portion of the curved parts are separated from straight parts and undergo their own alignment, while the remaining curved parts, probably due to sterical reasons, may be entangled (Figure 3). This case of hybrid microtubules illustrates how strong are spontaneous tendencies for segregation of morphologically different microtubule parts (straight from curved parts) while

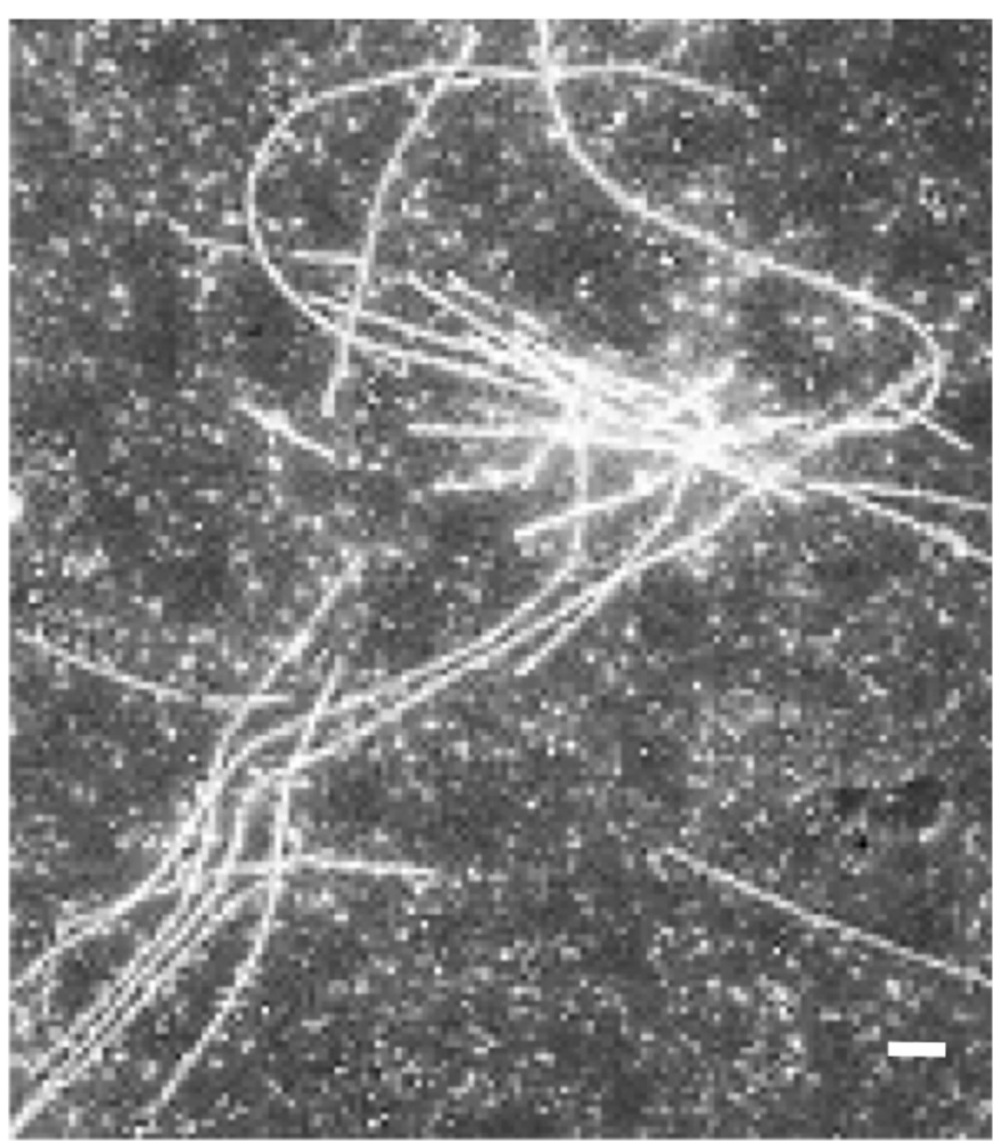

Figure 3 The segregation ( demix ) and alignment of straight and curved parts of hybrid microtubules, on the basis of morphological criteria. Hybrid microtubule is composed of morphologically different regions: straight and curved. Morphologically different regions tend to be spontaneously segregated along the same microtubule, while morphologically corresponding regions tend to be aligned between the neighboring hybrid microtubules. Bar is $0.1 \mu \mathrm{m}$. 
there is also strong isomorphic alignment (curved with curved and straight with straight parts).

Since the curved microtubules were minority in our experiments their effects on the direction of self-organization process could be negligible. However, we estimated the average curvature by applying circle approximation. Therefore we have calculated the curvature as inverse of relevant circle radius. The obtained average curvature of the curved microtubules, which are a minority in the straight microtubules dominated fiber (Figure 1A), is $0.001 ? 0.00039 \mathrm{~nm}^{-1}$, while the obtained value of curvature for the curved microtubules outside the fiber is $0.006 ? 0.0008 \mathrm{~nm}^{-1}$. Therefore, in the straight microtubules dominated fiber, the average curvature of the curved microtubules (minority) is smaller by factor 6 than that of the non-ordered microtubules, and by factor 1.5 than the curvature of the curved microtubules of curved microtubules dominated fiber, as shown in Figure 2A.

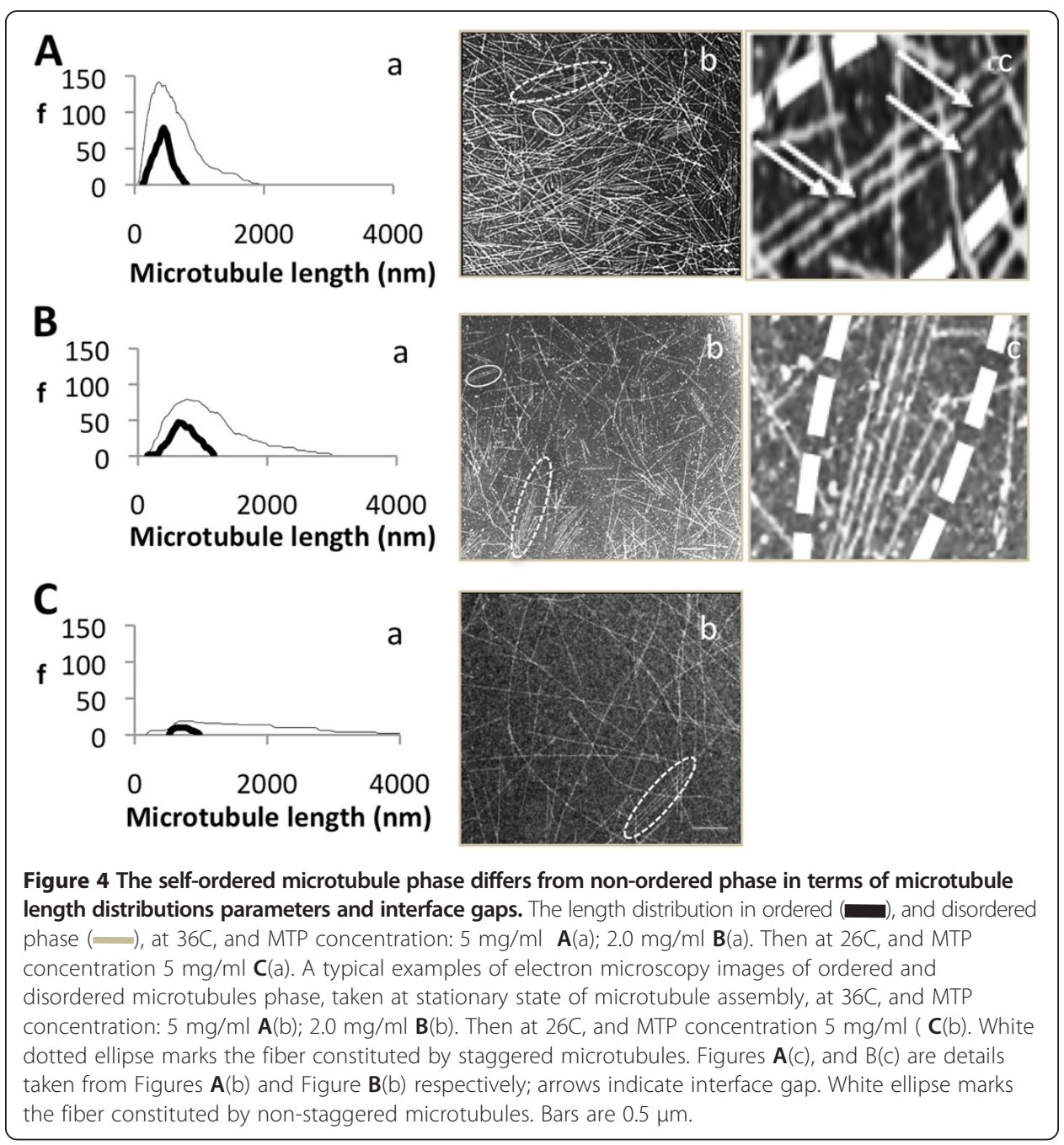


The basic topological features of straight microtubule fibers

Our results show that microtubule fiber patterns, in vitro, manifest in two configurations: axially shifted (staggered) and non-shifted microtubules. Fibers consisting of axially shifted microtubules are shown in Figure 1A, Figure 4A(b), B(b) and C(b) (white dotted ellipse). The pattern consisting of non-shifted neighboring microtubules is visible in Figure $4 \mathrm{~A}(\mathrm{~b})$ and $\mathrm{B}(\mathrm{b})$ (white ellipse). The fiber consisting of non-shifted neighboring microtubules cannot be extended.

The axial microtubule shift may extend fibers for several average microtubule lengths. Microtubules that constitute the extended fiber are laterally separated by microtubuleassociated proteins (MAPs) by approximately $15 \mathrm{~nm}$, while they are axially separated by irregular nanometer size microtubule free spaces i.e. the interface gaps. The interface gaps have irregular lengths that on average are commensurate to the average lengths of MAPs.

There are numerous interface gaps randomly located throughout the fiber. As mentioned, the size of the interface gap varies from location to location across the microtubule fiber but is always at a nanometer scale (Figure $4 A(b), B(b), C(b)$ ). The interface gap is laterally semi-confined by the walls of neighboring (parallel) microtubules and thus, is partially and unevenly separated from the bulk solution. The variability of the size of the interface gap is determined by the growth/shrinkage stage of microtubules that constitute the axial semi-confinement.

\section{Fiber imposes topological restrictions onto its constitutive microtubules}

The length distribution of microtubule self-organization into a fiber is narrower than that of microtubules outside the fiber

In this section, we compare the distribution of microtubule length in the ordered versus non-ordered phase (Figure 3). The exemplars of typical electron microscopy images of ordered and non-ordered microtubules have been obtained during a stationary state of microtubule assembly, at 36?C and MTP concentration of $5.0 \mathrm{mg} / \mathrm{ml}$ (Figure $3 \mathrm{~A}(\mathrm{~b})$ ) and $2.0 \mathrm{mg} / \mathrm{ml}$ (Figure $3 \mathrm{~B}(\mathrm{~b})$ ) as well as at 26 ? C and a MTP concentration of $5.0 \mathrm{mg} / \mathrm{ml}$ (Figure $3 \mathrm{C}(\mathrm{b})$ ).

Different degrees of microtubule self-organization (doublets, triplets etc.) are seen at both temperatures (36? C and 26?C) and at concentrations of MTP above $1.0 \mathrm{mg} / \mathrm{ml}$. Statistical parameters of these data are shown in Figure 5A. The average microtubule length increased as the concentration of MTP decreased. The average length dramatically increased at a lower temperature (26?C) (at the MTP concentration of $5.0 \mathrm{mg} / \mathrm{ml}$ ).

When we compared length distributions of ordered versus non-ordered microtubules (Figure $4 \mathrm{~A}(\mathrm{a}), \mathrm{B}(\mathrm{a}), \mathrm{C}(\mathrm{a})$ and Figure $5 \mathrm{~A})$, we obtained interesting results. The length distribution of ordered microtubules was always significantly narrower than that of non-ordered microtubules. In case of an MTP concentration of $5.0 \mathrm{mg} / \mathrm{ml}$, the length distribution of ordered microtubules was approximately 3 -fold narrower than that of non-ordered microtubules. In case of an MTP concentration of $2.0 \mathrm{mg} / \mathrm{ml}$, the length distribution of ordered microtubules is approximately 2.5 times narrower than that non-ordered microtubules. When the temperature was decreased to 26 ? $\mathrm{C}$ and at a concentration of $5.0 \mathrm{mg} / \mathrm{ml} \mathrm{MTP}$, the length distribution of ordered microtubules was approximately 9-fold narrower than that of non-ordered microtubules. 


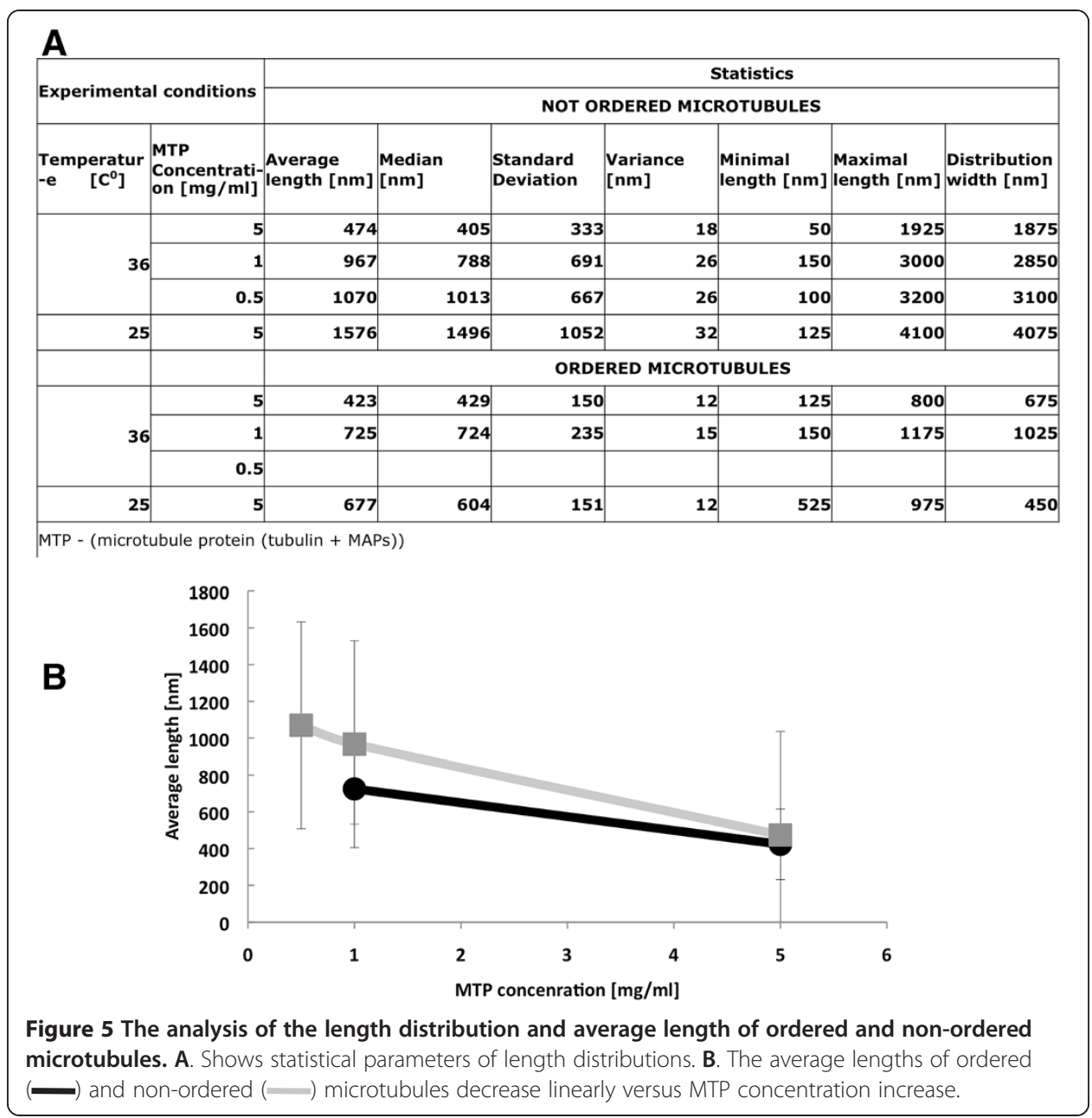

The average length of self-organized microtubules in a fiber is always shorter than those outside a fiber

The average length of self-organized microtubules in a fiber is 1.12?, 1.33? and 2.33? shorter than the average length of non-organized microtubules at $5.0 \mathrm{mg} / \mathrm{ml} \mathrm{MTP}$ and 2.0 $\mathrm{mg} / \mathrm{ml} \mathrm{MTP}$ at 36 ? C and $5.0 \mathrm{mg} / \mathrm{ml} \mathrm{MTP}$ at 26? C respectively (Figure $5 \mathrm{~A}$ ).

The average length of both microtubule populations decreases linearly versus MTP concentration see graph in Figure 5B. It is interesting to note that the range of standard deviation see graph in Figure 5B, and the related variance, see Figure 5A of ordered microtubules inside the fiber is much more narrower than that of the non-ordered microtubules outside the fiber.

\section{The total number of microtubules that constitute an individual fiber is always restricted}

Under our experimental conditions, we found that fibers comprising non-shifted microtubules consisted of approximately $\sim 2$ to $\sim 5$ microtubules (Figure $4 \mathrm{~A}(\mathrm{~b}), \mathrm{B}(\mathrm{b})$ ). However, fibers comprising axially shifted microtubules consisted of a minimum of 2 and a maximum of 12 microtubules (Figure 1A, Figure 4A(b), B(b), C(b)). 


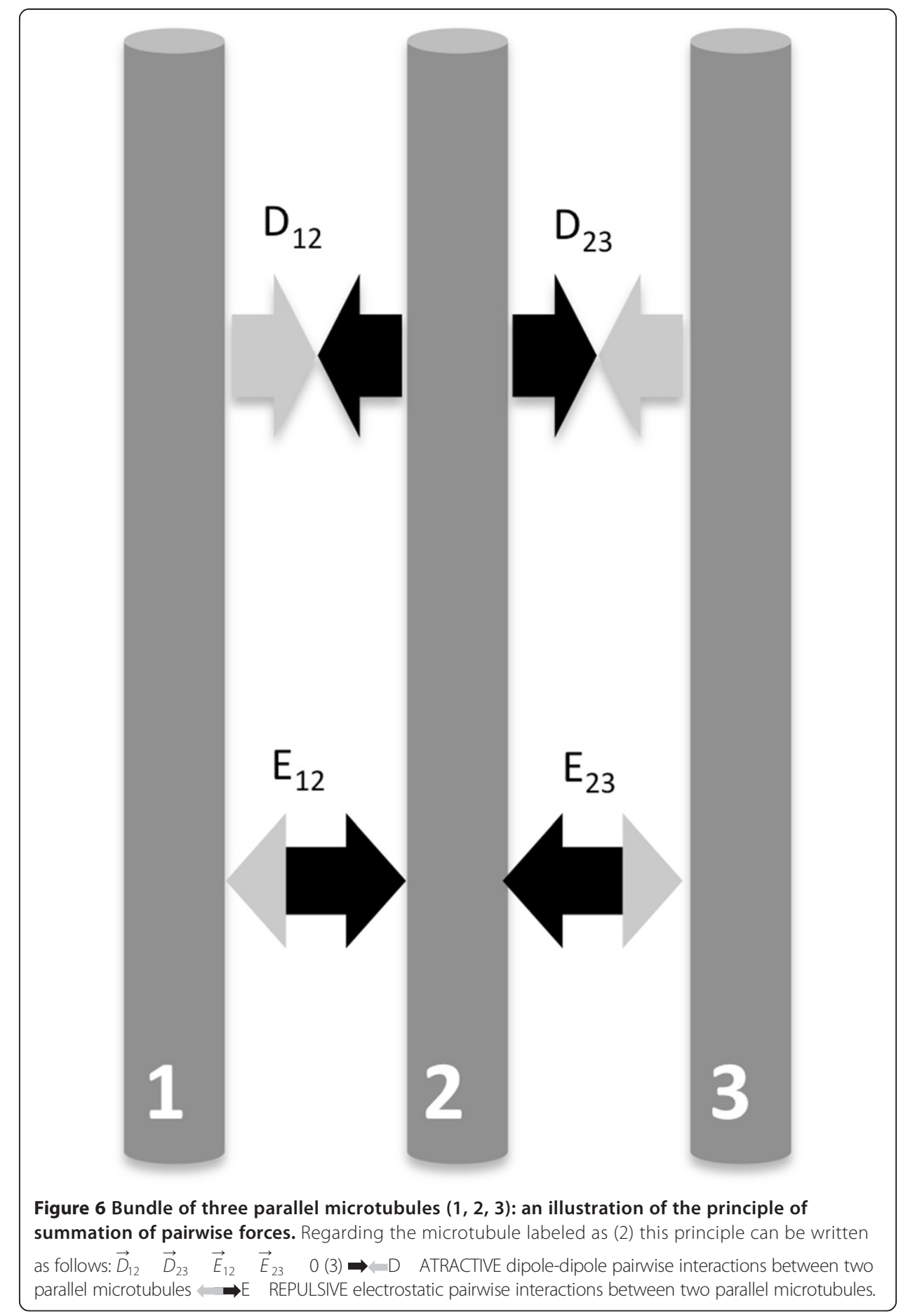

The pairwise electrostatic forces follow the principle of summation under the crowded conditions in our experiments

Once entropically brought close enough (such as in Figure 1A, Figure 2A, Figure 3, Figure $4 \mathrm{~A}(\mathrm{~b}), \mathrm{B}(\mathrm{b}), \mathrm{C}(\mathrm{b}))$ straight microtubules or straight part of curved microtubules may fall within the range of action of a variety of pairwise electrostatic forces. Due to their principle of summation these forces further tune ordering of pattern, increase its 
stability and endow constituting microtubules with cooperativeness as illustrated through the following text and in Figure 6; eq. 3. The microtubule related electrostatic forces have two sources from which they originate: (a) highly charged $\alpha \beta$-tubulin which in addition possesses permanent dipole moment [39,40], and (b) salty (aqueous based) medium which is necessary for microtubule growth (in vitro as well as in vivo). Salt in aqueous medium is dissolved into its constituting ions and this concentration is represented in terms of ionic strength. As far as ionic strength is concerned microtubules undergo spatial self-ordering in the range of physiological ionic strength $(0.1-0.2 \mathrm{M})$ and somewhat lower $[35,41]$. Finally, in order to have as much as possible satisfactorily picture about interactions which guide the microtubules spatial pattern formation in our microtubule system, it is important to bear in mind that: (a) ionic strength was $\sim 0.1 \mathrm{M}$, and (b) $\alpha \beta$-tubulin concentration was above the threshold considered as critical for microtubule crowded conditions. Under these solution conditions, variety of interactions may occur in the system, but microtubules self-organization in particular may be affected mainly due to: microtubule surface charge (repulsive electrostatic interactions), dipole-dipole attractive interactions between two neighboring microtubules, screening microtubules negative charges by salt counterions, charge-fluctuation forces between microtubules, and the principle of summation of pairwise electrostatic interactions. However, under conditions of our experiments (salt concentration $0.1 \mathrm{M}$ ), charge-fluctuations interactions are at least about an order of magnitude smaller than electrostatic interactions [30]. Therefore, we continue our analysis having in mind that electrostatic repulsive interactions and dipole-dipole attractive interactions are critical in ruling the formation and maintenance of microtubule pattern under our experimental conditions.

Let us now consider interactions which straight microtubules experience by being organized in parallel fashion of spatio-temporal pattern, and immersed in salty medium. Due to negatively charged surface, the neighbouring microtubules will experience strong electrostatic repulsive force. This force is weakened significantly due to the screening with the salt counterions. It is calculated that if two straight parallel microtubules are at the axis-to-axis distance of $\sim 40 \mathrm{~nm}$, then the repulsive electrostatic force between them is about $\sim 9.0 \mathrm{pN}$ [42]. This calculation was made under the assumptions that: (a) the average microtubule length is $\sim 5 \mu \mathrm{m}$, (b) ionic strength of salty solution is $\sim 0.1 \mathrm{M}$ (therefore the effect of screening was included). Our experimental data show that at the same ionic strength $(\sim 0.1 \mathrm{M})$ bundled neighboring microtubules of average length of $5 \mu \mathrm{m}$ were kept approximately at the distance of $\sim 40 \mathrm{~nm}$. This obvious agreement between the theoretical predictions and our experimental data indicates per se that repulsive electrostatic force between charged microtubules is critical for bundle formation and maintenance. If so, then the immediate question is what is the force that may counterbalance this repulsive electrostatic force (force between the two charged microtubule)? It is quite natural to speculate that attractive dipole-dipole force is potential candidate to counterbalance this repulsive force. Under the similar solution conditions, mentioned above, dipole-dipole attractive interactions between the two neighboring microtubules were found to be about $\sim 330 \mathrm{pN}$, which per se is far stronger than repulsive electrostatic force [42]. Why then two neighboring microtubules, in the bundle, do not collapse onto each other? If this happens it would lead to a quite static structure. However, from the biological point of view, bundle has to be and it is indeed highly dynamic spatio-temporal pattern [27]. Fortunately, for microtubule in the bundle the situation is more complex than for two parallel microtubules 
outside of bundle. This is due to the principle of summation of pairwise forces, which is taking place under crowded conditions of our experiments. Indeed, because of a particular parallel geometry of bundled straight microtubules, the same kind of force may be self-attenuated. Let us illustrate how this principle may act in the case of a simple bundle constituted by three straight and parallel microtubules (Figure 6). In this case the microtubule, which is placed between its two exterior neighbors is continually under the influence of pairwise forces generated between it and its neighbors. In other words, the interior microtubule is in synchronous fashion pulled outward to the left and the right sides by dipole-dipole attractive forces. The net effect of summation of these pairwise forces taken together is their self-attenuation. On the other hand, the same microtubule is pushed inward to the left and the right directions by repulsive electrostatic forces generated by its exterior neighbours. Again, the principle of summation of pairwise repulsive forces will lead to their net self-attenuation. Obviously, the principle of summation of pairwise forces opens the ample possibilities of different combinations of forces that may support dynamic equilibrium of the microtubule self-organized pattern.

Let us see now if the principle of pairwise forces summation is working under conditions of our experiments. For that purpose we employed the criterion already developed in the work of [30]. The criterion is mathematically expressed in the following inequality

$$
n_{m}>>\frac{1}{b \pi\left(R^{2} a^{2}\right)}
$$

In the above inequality $\boldsymbol{n}_{\boldsymbol{m}}$ is the density of mobile ions in solution, $\boldsymbol{R}$ is the neighboring microtubules central axis-to-central axis distance, $\boldsymbol{a}$ is microtubule half diameter, and $\mathrm{b}$ is the length per unit charge along microtubule surface. For the salt concentration of $0.1 \mathrm{M}$ we obtain the following density of mobile ions

$\boldsymbol{n}_{\boldsymbol{m}} \quad 6.02 \times 10^{25} \mathrm{~m}^{-} 3$.

To calculate the right side of inequality (2) we use the microtubule distance $R=40 \mathrm{~nm}$, microtubule half-diameter $\boldsymbol{a}=12.5 \mathrm{~nm}$, and $\mathrm{b}=8$ ? . So for the right side of inequality (2) we obtain

$$
\frac{1}{b \pi\left(R^{2} \quad a^{2}\right)} \quad 0.00246 \times 10^{25} m^{-3}
$$

The left side of inequality (2) is far greater than its right side. Therefore criterion (2) is satisfied, and consequently the principle of pairwise forces summation is operating under our experimental conditions. Consequently, the principle of pairwise electrostatic forces summation leads to fine tuning (cooperation) of constituting microtubule motion within the fiber and its additional stabilization.

\section{The local GTP-tubulin depletion may occur in the immediate vicinity of the tips of microtubules}

The set of topological restrictions imposed on microtubules self-organized into a fiber is an important observation as it may indicate that an inherent mechanism regulates selfformation and maintenance of the fiber. It was shown that the depletion of GTP-tubulin in a region located in the immediate vicinity of the tips of microtubules might control the number of microtubules organized into a microtubule aster [43]. We assume that, similar 
to the case of a microtubule aster, local depletion of GTP-tubulin may be a part of local solution conditions that lead to a restricted number of microtubules that constitute a fiber.

Oddes calculations indicated that there are three factors that are critical in order to obtain total local depletion of GTP-tubulin near the tip of a single free (i.e. not bound in any spatial pattern) growing microtubule [44]. These factors are: the bulk GTP-tubulin concentration, the diffusivity and the rate of microtubule assembly. The calculations are based on a steadystate mean-field model for the concentration of GTP-tubulin and the assumption that there is only one GTP-tubulin binding site per microtubule that effectively contributes to microtubule assembly.

According to these calculations, total depletion of GTP-tubulin near the tip of a single microtubule may occur when the bulk GTP-tubulin concentration is $1 \mathrm{mg} / \mathrm{ml}$ (the threshold at which crowded conditions start to appear), diffusivity (intermediate) is $6.4 ? 10^{-12} \mathrm{~m}^{2} / \mathrm{s}$ and the assembly rate of an individual microtubule is $65 \mu \mathrm{m} / \mathrm{min}$ [44]. Experimentally measured, the real maximal microtubule assembly rate is $20 \mu \mathrm{m} / \mathrm{min}$ [45]. Odde therefore concluded that, under the given conditions, the GTP-tubulin depletion could not be created in front of a tip of a single growing individual microtubule.

The factors critical for total GTP-tubulin depletion in our experiments are different to those employed by Odde and favor the occurrence of depletion. The bulk GTP-tubulin concentration used in our experiments is approximately 10 times higher than those used by Odde. Lower GTP-tubulin diffusivity may favor the creation of GTP-tubulin depleted regions. Odde used an intermediate value for diffusivity. In our system, diffusivity is expected to be much lower due to a strong influence of boundary conditions imposed by the presence of a multitude of crowded, single microtubules as well as microtubule spatiotemporal structures (such as fibers). Therefore, the significantly higher degree of crowded conditions in our system may lead to the significantly lower diffusivity than an intermediate one employed by Odde [44].

Conversely, under highly crowded conditions (GTP-tubulin concentration approximately $7 \mathrm{mg} / \mathrm{ml}$ ), the local reaction diffusion conditions in front of a set of microtubule tips organized into a fiber may be quite different as compared to those of the single free microtubule, which in turn may favor the creation of small GTP-depleted regions in the immediate vicinity of the tips of microtubule fibers [46]. Let us assume that each individual microtubule (within a fiber) grows independently, so that we may assume that they independently take up GTP-tubulin from the common pool located in the immediate vicinity of their tips. According to Odde s calculations, a single free microtubule, in order to create a total local depletion of the GTP-tubulin, should grow at a rate of $65 \mu \mathrm{m} / \mathrm{min}$. Therefore, to reach the same degree of local depletion in front of the tips of a fiber comprising two microtubules, each microtubule should grow at an assembly rate of $32.5 \mu \mathrm{m} / \mathrm{min}$. Consequently, in a fiber consisting three microtubules, each microtubule should grow at a rate of $21.67 \mu \mathrm{m} / \mathrm{min}$ in order to produce a total local depletion (Figure 7).

If we accept Oddes assumption that there is only one GTP-tubulin binding site per microtubule, then (see the graph in Figure 7), total local depletion of the GTP-tubulin will certainly occur in close proximity of a fiber terminal, which is constituted by at least four microtubules (where the assembly rate of each microtubule is $16.25 \mu \mathrm{m} / \mathrm{min}$ ). However, in our experimental conditions, we found an abundance of fibers comprising only two and three microtubules (Figure 4), which may indicate that there is more than one GTP-tubulin binding site per individual microtubule that constitutes a fiber. Thus, under highly crowded 


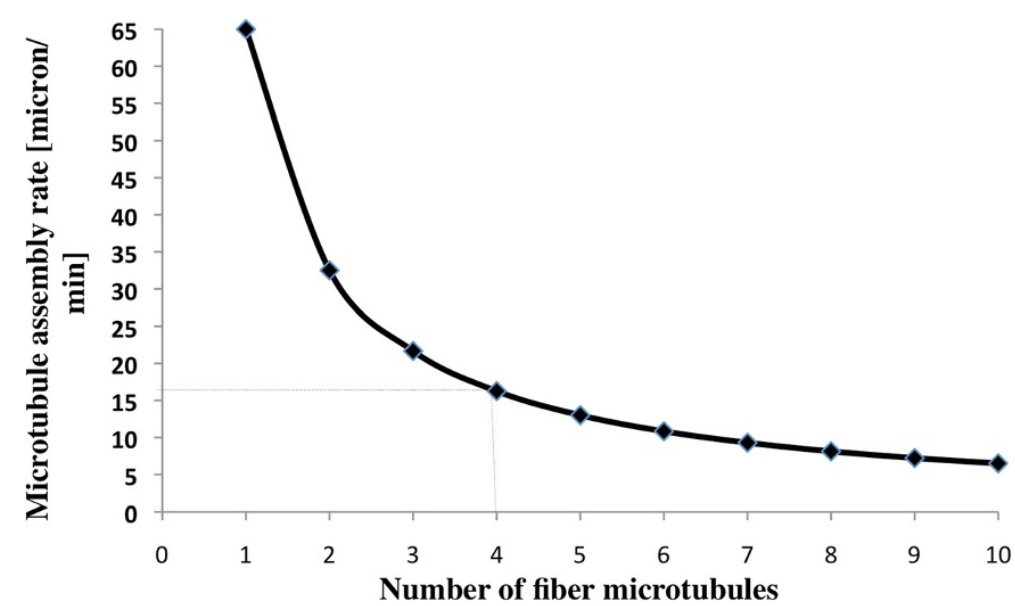

Figure 7 Theoretical estimation of effective assembly rate of each individual microtubule within a fiber at which the total depletion of GTP-tubulin in the immediate vicinity of fiber microtubule tips may be induced. At crowded conditions (see the text), the total local depletion of GTP-tubulin at the tip of individual, out of fiber, microtubule will occur if microtubule grow at 65 microns/min (Odde [44]). However, maximal microtubule growing rate is 20 microns/min (Shelden and Wadsworth [45]). Therefore total depletion of GTP-tubulin cannot be induced in front of individual, out of fiber, microtubule. However, each individual fiber microtubule independently contributes to the depletion. Since their effect on depletion is cumulative, they may induce depletion each growing at rate less than 65 microns/min. In the case of fiber constituted by the four microtubules, depletion can be induced. Indeed, if each of four microtubule grow at $\sim 16.5$ microns/min, then in terms of depletion efficiency; $16.54=66 \mathrm{microns} / \mathrm{min}$, total local depletion may be achieved. At number of fiber microtubules higher than four, depletion will be certainly induced.

conditions, a total local depletion may occur even if the fiber is made up by less than four microtubules (Figure 7).

The extension of the GTP-tubulin depletion zone versus the solution of blind mason problem

As we have mentioned above, apart from the tubulin concentration, its degree of freedom of motion manifested as translational and rotational diffusivity plays important role in the extension of GTP-tubulin depletion zone. In order to be docked in the appropriate location of GTP-cap tip the incoming tubulin emerges from the bulk solution. If emerging tubulin is endowed with full degree of freedom of motion, this would be the most favorable (energetically and entropically) situation. In other words, its subsequent manoeuvres and placement in the location with any orientation would be equally probable. Therefore, the blind mason problem would be directly solvable. However, crowded conditions, depending of their degree of crowdedness, may impair full freedom of tubulin motion by imposing anisotropic conditions.

Consequently this may impair the solution of blind mason problem. Nominally, we are working at and above tubulin concentration of $1 \mathrm{mg} / \mathrm{ml}$. This concentration is considered as threshold above which crowded conditions arise [43,47-49]. A propos of blind mason, it is reasonable to ask the following question: does tubulin has enough space to perform necessary manoeuvres by means of free rotation and translation at the critical concentration of microtubule self-organization and higher? As long as incoming tubulin has enough space to perform free rotation and translation, the problem of blind mason is solved. Concerning tubulin, as far as we know, there are no appropriate data that can indicate the 
changes (reduction) of degree of freedom of motion versus crowded conditions. However, recently it was studied the emergency of anisotropy of motion of lysosome versus its concentration within the range which includes crowded conditions (Table three in [28]). The volume of lysozyme molecule, approximated by cylinder is $\sim 141.3 \mathrm{~nm}^{3}$ [50]. The volume of $\alpha \beta$-tubulin molecule, approximated by cylinder is $\sim 464.5 \mathrm{~nm}^{3}$ [51]. To extrapolate the observed data of emergency of anisotropy of lysozyme motion and apply it to the case of tubulin, we calculate the accommodating space factor for both lysozyme and $\alpha \beta$-tubulin. The term accommodating space factor refers to the number of times the accommodating space is greater than the volume of the protein. Accommodating space of protein is the space, which accommodates protein free rotation and translation. The results (Table 1) show that anisotropy of lysozyme motion emerged at highly crowded condition, i.e. at lysozyme concentration of $150 \mathrm{mg} / \mathrm{ml}$. At that concentration the accommodation space factor for lysozyme is 1.12 . Since at the same concentration, the accommodating space factor for tubulin (2.61) is very close to the lysozymes accommodating space factor, we speculate that anisotropy of tubulin motion should emerge at approximately close concentration of lysozymes one. Therefore, in our working concentration range $(1-10 \mathrm{mg} / \mathrm{ml})$ crowded conditions are too weak to impair overcoming blind mason problem. From the dynamical point of view, in order to perform subsequent positional-orientational adjustments by means of translational and rotational diffusion tubulin is endowed with additional local space - this will be done at the cost of depletion zone extension.

\section{Mutual interdependence of microtubule pattern and associated local solution conditions}

Through all our data we see that the spatio-temporal pattern of microtubules are a sensitive function of solution conditions. For example in the presence of excess calcium, curved microtubules and therefore curved fibers are formed (Figure 2A,B; Figure 3). Furthermore, the concentration of GTP-tubulin affects parameters of microtubule selforganization. The occurrence of fibers is more probable at more crowded conditions. The length distribution of patterned microtubules changes differently in higher GTP-tubulin concentrations compared to those of non-patterned microtubules. In the presence of high GTP-tubulin concentrations, the width of the length distribution of patterned microtubules is shorter compared to those of non-patterned microtubules (Figure 4A,B,C).

Our calculations have shown that total local depletion of GTP-tubulin may occur in the immediate vicinity of microtubule tips due to pattern growth dynamics (Figure 7).

Table 1 Emergency of anisotropy of a $\beta$-tubulin molecule motion in the bulk solution

\begin{tabular}{|c|c|c|c|c|}
\hline \multirow[b]{2}{*}{ Concentration $[\mathrm{mg} / \mathrm{ml}]$} & \multicolumn{2}{|c|}{ LYSOZYME } & \multicolumn{2}{|c|}{ TUBULIN } \\
\hline & Fas & Anisotropy & Fas & *Anisotropy \\
\hline 1 & 169.85 & $\mathrm{NO}$ & 394 & $\mathrm{NO}$ \\
\hline 10 & 16.99 & $\mathrm{NO}$ & 39.4 & $\mathrm{NO}$ \\
\hline 150 & 1.12 & YES & 2.61 & YES \\
\hline
\end{tabular}

The emergency of anisotropy of lysozyme molecule motion may qualitatively indicate the emergency of anisotropy of a $\beta$-tubulin motion under corresponding solution conditions. $F_{a s}$ is the accommodating space factor which says how many times the accommodating space is greater than the volume of one protein molecule. Accommodating space is the space which accommodates free rotation and translation of single protein molecule. The volume of lysozyme molecule, approximated by cylinder is $\sim 141.3 \mathrm{~nm}^{3}$ (Matthews and Remington [50]). The volume of a $\beta$-tubulin molecule, approximated by cylinder is $\sim 464.5 \mathrm{~nm}^{3}$ (Nogales, et al. [51]). *Anisotropy is the anisotropy of a $\beta$-tubulin motion obtained by extrapolation from lysosyme data under corresponding solution conditions (Table three in Dlugosz and Antosiewicz [24]). 


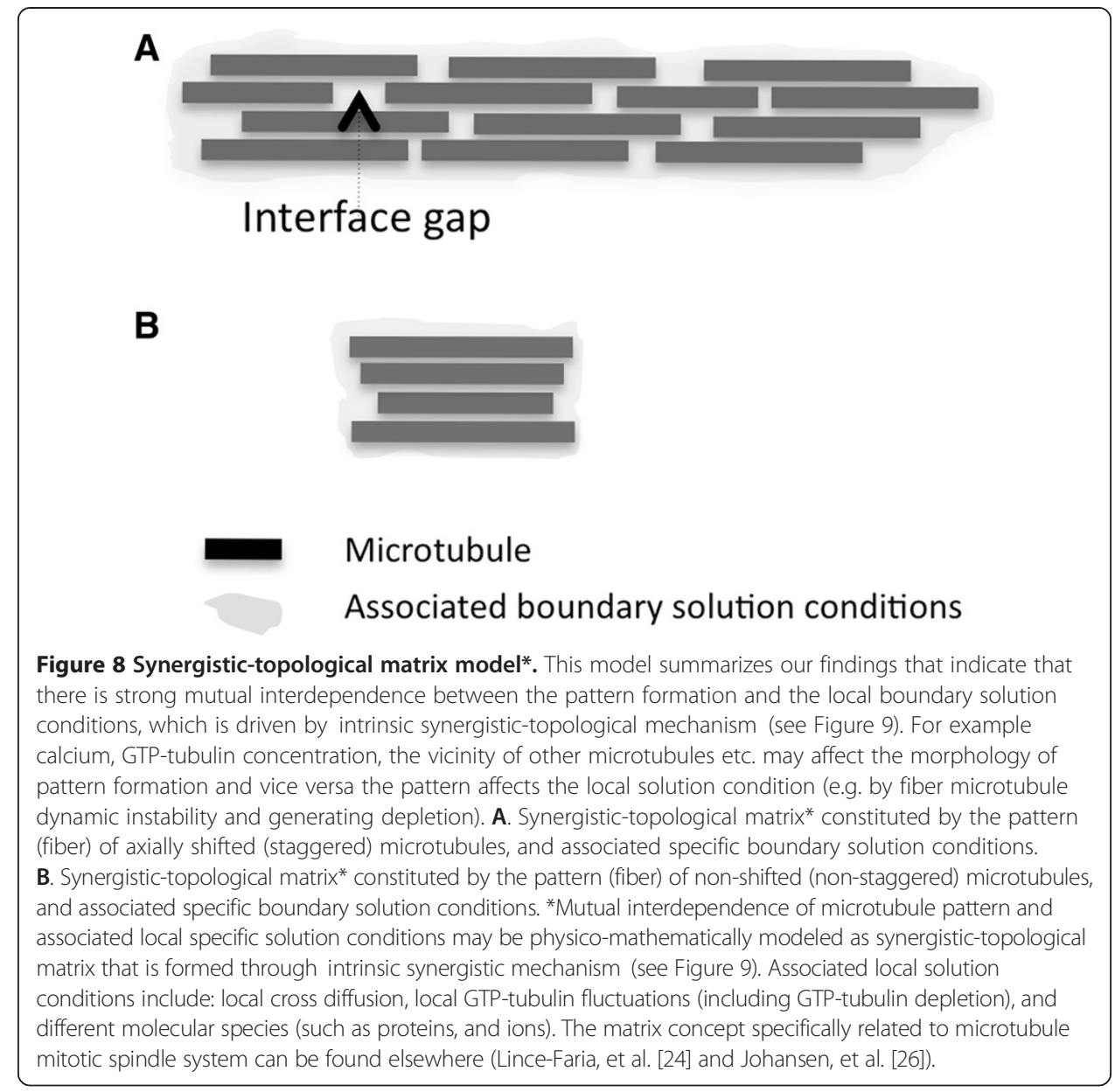

Therefore, collectively, these data indicate that there is strong mutual interdependence between the pattern morphology dynamics and local solution conditions that can be physico-mathematically modeled by a matrix that is driven by intrinsic synergistictopological mechanisms (Figure 8A,B; Figure 9A,B,C).

\section{Discussion}

Existing data indicate that synergism is an essential element involved in biological complexity of cytoskeletal (including microtubules) function. However, the mechanism(s) by which synergism guides the self-organization of the microtubule system is yet to be understood. For this reason it would be useful to study synergistic mechanism(s) related to microtubule multitudes under minimalistic but crowded (in terms of GTP-tubulin concentration) biological conditions, in vitro.

\section{Entropically driven spontaneous demix and self-organization of curved and straight microtubules}

We have observed microtubule self-organization under crowded conditions where straight microtubules were dominant. However, to get rationale for this particular case it is helpful to bear in mind what happen if the concentration numbers of straight and 


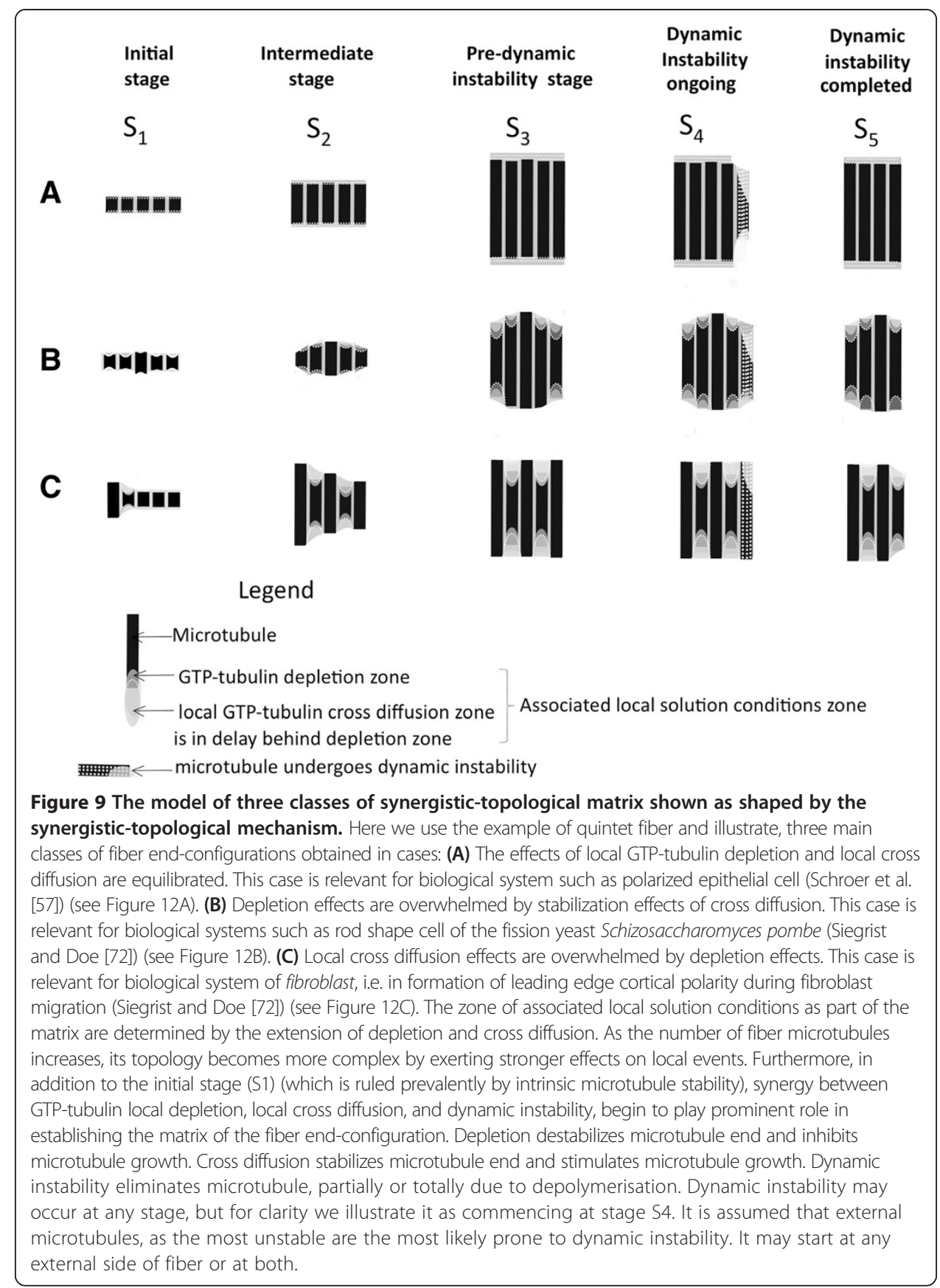

curved microtubules are approximately equal. In addition, the system contains minority of microtubules whose curvature is at the critical threshold, which enables them to undergo alignment (we call these microtubules slightly curved).

If so, then at sufficiently crowded conditions, the system is unstable and undergoes spontaneous phase segregation (demix) of coexisting phases, while slightly curved microtubules join the population of straight microtubules [52]. Also when the straight microtubules become much longer than the distance between neighboring microtubules, then the spontaneous orientational ordering is initiated [53]. At the end of the process (composed of demix and orientational ordering) the total gain of entropy for straight, 


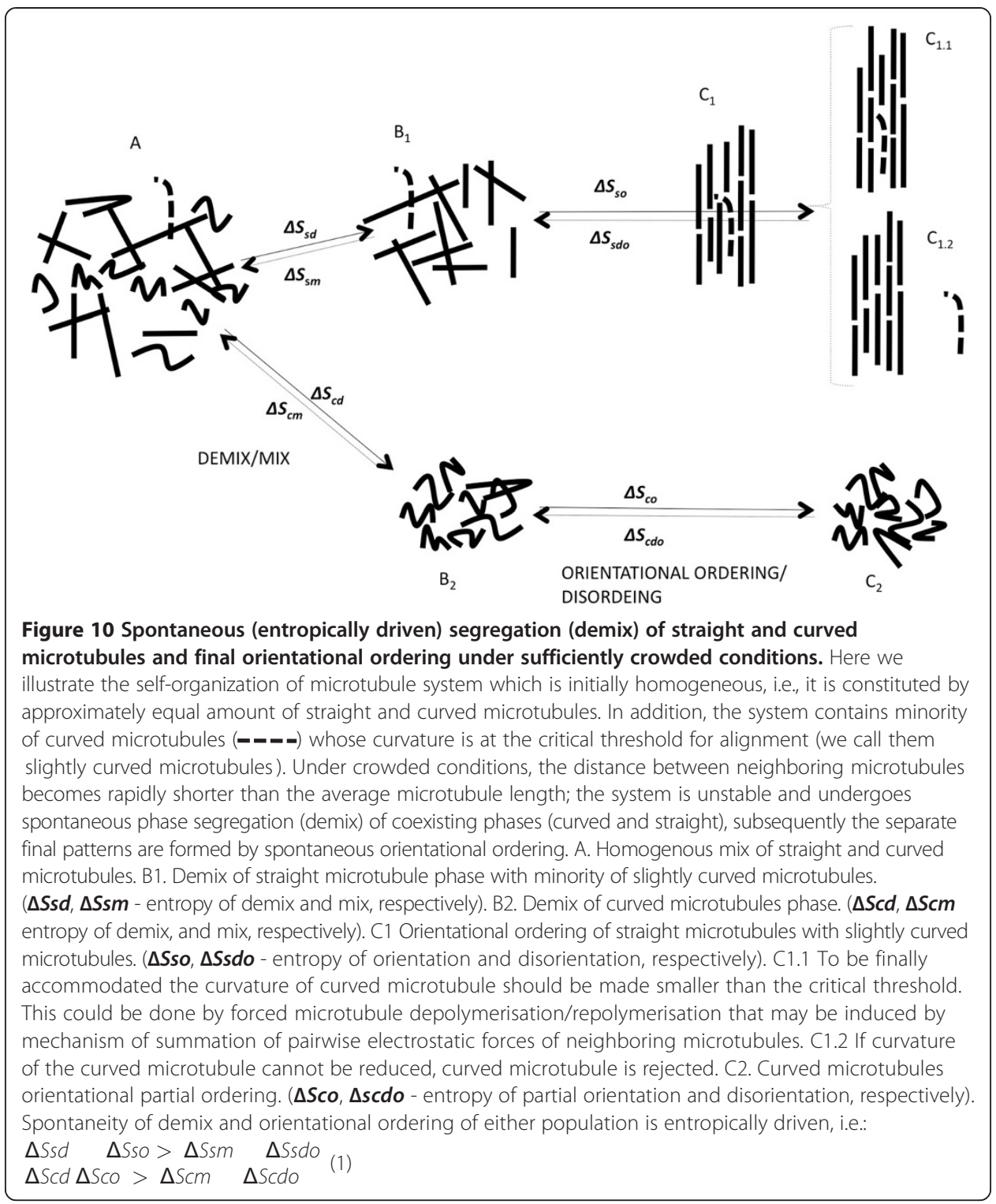

curved, and slightly curved microtubule populations will be higher than the total loss of entropy during mix and orientational disordering (Figure 10A, B1, B2, C1, C2; inequality (1)) $[52,53]$. Therefore, the total process constituted of phase demix and final pattern formation due to orientational ordering, is entropically driven.

In practice slightly curved and straight microtubules can be often mixed in the self-organized pattern, such as the fiber in Figure 1A. Here, the main question is about the mechanism how the slightly curved microtubule got into the fiber structured of dominantly straight microtubules? We have no data regarding the direct history of how the slightly curved microtubule got into the fiber (Figure 1A). However, the data regarding the average curvature indicate that the average curvature of non-ordered microtubules is greater than the curvature of slightly curved microtubules built in ordered structure of the fiber. We can speculate that there are two possible scenarios. The first scenario is that the entropically driven processes of demix and alignment may temporarily bring slightly curved microtubule into the fiber structure (Figure 10C1). Once 
microtubules have been brought close enough to each other by entropical drive making (loose) fiber (Figure 10C1), the pairwise electrostatic forces in particular the action principle of pairwise forces summation further tune and stabilize the fiber [30,39,40,42]. Consequently, the curvature of a slightly curved microtubules may become even smaller due to the complex neighboring microtubule electrostatic interactions within the fiber, in particular due to the actualization of the principle of summation of pairwise electrostatic forces which may cause the cycle of microtubule disassembly/reassembly and/or their direct bending. Therefore, the slightly curved microtubule may be straightened and stabilized i.e. accommodated as part of fiber structure (Figure 10C1.1).

The second possible scenario is that the fiber structure may simply reject microtubules whose curvature is above the certain threshold as energetically and entropically costly in order to be accommodated within the fiber structure (Figure 10C1.2).

Concerning segregation and alignment phenomena in the system of hybrid microtubules (Figure 3) one can explain it on the basis of Onsager s principle: curved microtubules, with the certain degree of straightness and rigidity, undergo partial parallel ordering and in part just entanglement [53] (Figure 3).

\section{Spontaneous symmetry breaking during microtubule fiber formation and its network self-formation provides evidence for the presence of intrinsic synergy}

Although simple, our chosen system enables spontaneous formation of different forms of microtubule fibers comprised of straight and curved microtubules, which further undergo spontaneous segregation and symmetry breaking, thereby forming more complex spatial networks (Figure 1A and Figure 2A; Figure 1B, and Figure 2B). Symmetry breaking is obviously accompanied by an abundance of spatially and temporally correlated microtubule multitudes. Therefore, spontaneous symmetry breaking during microtubule self-organization reflects the presence of intrinsic synergy mechanism(s) $[37,38]$.

\section{The fiber imposes topological restrictions on constituting microtubules}

An exemplar of different classes of fibers formed under different solution conditions are illustrated in Figure $4 \mathrm{~A}(\mathrm{~b}), \mathrm{B}(\mathrm{b})$, and $\mathrm{C}(\mathrm{b})$ and marked by a dotted white ellipse. We have found that under a given solution condition the self-organization of a microtubule fiber imposes a set of topological restrictions onto microtubules that constitute the fiber. We have found variable, nanometer-size microtubule free spaces located between fiber microtubules that are approaching/receding (i.e. tip-to-tip), along the same fiber track (Figure $4 \mathrm{~A}(\mathrm{c})$ ), $\mathrm{B}(\mathrm{c})$, see also an illustration of the interface gap in Figure 8A). As already mentioned, we termed these nano-spaces interface gaps. The nanometer-size microtubule free spaces, between tip-to-tip fiber microtubules are also observed in a microtubule MAPs free system, where fibers are ordered by strong external magnetic fields [54]. In our system, MAPs are present and fibers are spontaneously self-organized. It was also shown that microtubules undergo spontaneous self-organization in the form of liquid nematic crystals under solution conditions commensurable with ours [55]. Importantly, the pattern of nematic liquid crystals implicates the existence of microtubule free spaces between tip-to-tip of axially approaching microtubules. The microtubule free spaces are also present in the fibers of axonal microtubules that have an axially shifted configuration [56,57]. Therefore, the presence of the microtubule interface gap, under a variety of experimental conditions, may 
perhaps suggest that it has a regulatory role in events that may be transmitted along individual fiber microtubules such as mechanical force, energy and information processing [26,39,58-61]. This may be critical in cellular wound healing [62,63].

In relation to topological restrictions in fibers, the following are pertinent: (a) the total number of microtubules that constitute a fiber is always restricted at given solution conditions; (b) the average length of microtubules within a fiber is consistently shorter than the average length of free microtubules; (c) the length distribution of fiber microtubules is significantly narrower in comparison to free microtubules (Figure $4 \mathrm{~A}(\mathrm{a}), \mathrm{B}(\mathrm{a}), \mathrm{C}(\mathrm{a})$ ). The associated numerical data are summarized in Figure 4A,B. In addition, we believe that the differences in standard deviations between ordered and non-ordered microtubules as shown in Figure 5B graph, and the differences in variance intervals (Figure 5A) may come, not from the quality of measurement, but from a different nature (in terms of freedom of motion) of these two microtubules populations. Non-ordered microtubules grow freely, stochastically and independently of each other, while ordered microtubules are subjected to the local structural packing constriction conditions, which may prevent significant differences in their lengths. In our view, these differences in standard deviations and variances, as such may per se indicate that particular mechanism, that is, synergy mechanism may be behind the microtubules self-ordering event.

By being inherent to the fiber microtubule topology, these restrictions drive the fiber self-organization. To explain how these restrictions function, we propose the existence of an intrinsic synergistic-topological mechanism.

\section{The synergistic-topological mechanism}

It is very well known that microtubules capacity to undergo self-ordering is critical for their biological function. Due to its biological function, a stable self-ordered microtubule pattern has also to retain a certain degree of freedom of motion (flexibility). This extraordinary dynamic feature of microtubule self-organized patterns is realized by means of the principle of summation of pairwise electrostatic forces [30]. This principle plays fiber integrative role, and in the same time it endows constituting microtubules with the certain degree of cooperativeness. Once entropically brought close enough (such as in Figure 1A, Figure 2A, Figure 3; Figure 4A(b), B(b), C(b)) straight microtubules or straight part of the curved microtubules may fall into the range of action of a complex variety of pairwise electrostatic forces. While these forces stabilize the pattern, they also, due to the principle of their summation, endow constituting microtubules with certain degree of cooperative motion (Figure 6). This is of essential biological importance. We have shown that under our experimental crowded conditions the principle of summation of pairwise forces is working (inequality (2) is satisfied). Although it is not limited to the triplet of microtubules, the principle of forces summation can be fully understood in the case of microtubules triplet (Figure 6 eq. (3)). Indeed, left neighbor (labeled with number 1) of intermediate microtubule (labeled with 2) knows what exactly does its right neighbor (labeled as 3), and responds with appropriate reaction according to eq. (3). This is the essence of elementary cooperativeness among fiber constituting microtubules. Rooted in the principle of summation of pairwise electrostatic forces, the cooperativeness of fiber constituting microtubules is an important factor in the synergistic-topological mechanism about which we will talk later. 
Substantial differences between the topology of self-organized microtubules (where the principle of summation of pairwise forces is working) and free single microtubules, under the same bulk solution conditions, suggest that some local solution factor(s) and mechanism(s) may be responsible for the above-mentioned topological restrictions in fiber microtubules. Based on the analysis of our data, we propose that the key solution factor responsible for the set of the above-mentioned topological restrictions in fiber microtubules is the local depletion of GTP-tubulin. While depletion occurs locally in the immediate vicinity of the tips of fiber microtubules, and therefore acts on their dynamic directly, the principle of summation of pairwise electrostatic forces operates between neighboring microtubules all along their lengths - across the fiber (Figure 6).

It was shown that the local depletion of GTP-tubulin cannot occur in the immediate vicinity of the tip of only one (single) free growing microtubule [44]. Our calculations revealed that at given solution conditions the local depletion of the GTP-tubulin may occur in the immediate vicinity of the tips of multitude of self-organized fiber microtubules at a certain critical number of fiber microtubules (Figure 6).

The question that arises from this is what would be the mechanism by which local GTP-tubulin depletion may operate to control the size of microtubule fiber? During fiber growth each fiber microtubule grows independently. Therefore, the tips of all fibers constituting microtubules are independently taking up GTP-tubulin from the same small pool that is located in the immediate vicinity of fiber terminals, that is, within a nanometer distance from the tips of growing/shrinking microtubules. Consequently, all of the microtubule tips from the same fiber may strongly compete for incoming GTPtubulin. The competition intensifies with increased number of growing microtubules within the fiber. Each individual fiber microtubule contributes independently to the eventual local GTP-tubulin depletion, as each microtubule, of the same fiber, grows independently. Therefore, we may assume that the total contribution to depletion may be calculated as a linear sum of all individual fiber microtubule contributions, sometimes referred to as collective reactivity [64]. If the number of growing microtubules, which constitute a fiber, is high enough, then they may take up more GTP-tubulin from the pool than can be supplied by local diffusion. Therefore, local depletion of the GTPtubulin may effectively and instantly occur in the immediate vicinity of a growing fiber terminal (Figure 6).

The local GTP-tubulin depletion may randomly and non-uniformly lead to the exhaustion of the addition of GTP-tubulin to the tips of different individual microtubules and thus, may randomly slow down or even halt the growth of some individual fiber microtubules. The instant halting of the growth of an individual fiber microtubule, in conjunction with the eventual burst of GTP-hydrolysis at the terminal GTP-tubulin subunits of that particular microtubule, creates conditions that instantaneously switch on dynamic instability of the same fiber microtubule [65]. Once the dynamic instability is switched on, the microtubule may shrink, or it may be catastrophically and completely destroyed. In this way the number of growing fiber microtubules may be reduced due to dynamic instability in conjunction with the dynamic of depletion impact.

Once the number of growing microtubules is reduced, the depletion may start to decline due to a constant supply of fresh GTP-tubulin by local cross diffusion, which is efficiently empowered by crowded conditions. In this way, the growth of some of fiber microtubules, whose growth was stopped due to depletion, may resume again while the 
others may continue to grow at an optimal rate according to the given solution conditions. The spatial variation in local GTP-tubulin depletion can be understood as the nonthermal and nonequilibrium GTP-tubulin local fluctuations [66,67]. Under the given conditions, these fluctuations may be maintained as long as they are coupled to dynamic instability and cross diffusion of GTP-tubulin. This tight cooperation between dynamic instability, GTP-tubulin local fluctuations and cross diffusion, is the essence of synergy mechanisms, which operate at microtubule tips of the fiber terminals.

Finally, the action of the principle of pairwise forces summation is constantly implicated in all of these events, and can be understood as essential part of a synergy mechanism, that is its modulator.

Apart from the apparent randomness of local GTP-tubulin depletion, there are two stereo-specific aspects of a microtubule GTP-cap tip that may topologically condition the rate of GTP-tubulin depletion, and the extension of GTP-tubulin depletion zone. The first of these is the burst of GTP-hydrolysis at the microtubule GTP-cap tip, and the second is related to the necessity of blind mason problem solving.

\section{The burst of GTP-tubulin hydrolysis}

The burst of GTP-tubulin hydrolysis is topologically conditioned by intrinsic structural parameters of the GTP-cap of each individual fiber microtubule [65]. In turn, the GTP-hydrolysis burst may switch on dynamic instability of a particular individual microtubule and cause its subsequent partial or total depolymerization. Therefore, under the given solution conditions, the elimination pattern of an individual fiber microtubule by dynamic instability is topologically conditioned by intrinsic GTP-cap stereo-selective parameters. This stereo-selectivity may lead to different end-configuration topologies of fiber terminals (Figure 9A,B,C).

GTP-tubulin depletion zone is conditioned by the necessity of blind mason problem solving It is well documented that tubulins structural arrangement in any microtubule lattice (known so far) strictly follows unique symmetry pattern manifested as the left-handed (chirality) helicity [68-70]. It was proposed that since tubulin, as the main building block of microtubule wall, possesses well defined particular asymmetric shape, it can be docked with precision by appropriate packing constraints of symmetry pattern of microtubule lattice, and this should be the most direct and the easiest way to solve the problem of blind mason during microtubule growth [29]. Therefore in dynamic picture tubulin docking needs additional effective local space to perform necessary spatial adjustments due to rotational and translational movement. In turn, this will condition the size of the GTP-tubulin depletion zone. We believe that the proper solution to the blind mason problem lies within the frame of nonlinear dynamic. Before its docking begins in the proper location of GTP-cap tip lattice, the incoming tubulin has to emerge from the bulk solution. In order to initiate and complete the docking, it is obviously preferable (energetically and entropically) for tubulin to have on disposition the full degree of freedom of its rotational and translational motion. In this way, all the subsequent positional and orientational adjustments will be almost equally possible. Anisotropy in tubulin motion occurs due to crowdedness, and in turn it (anisotropy) may impair, energetically and entropically, the tubulin docking. 
Consequently, in this situation the capacity to overcome the problem of blind mason is reduced. However, by extrapolating data for lysosyme, we have shown in Figure 7 that in vitro experiments, where only tubulin is present in the solution, anisotropy in tubulin motion in the bulk may appear only under the strong crowded conditions (the bulk concentration of tubulin $\geq 150 \mathrm{mg} / \mathrm{ml}$ ) [28]. Therefore, in our experiments, the degree of crowdedness is enough low to allow the problem of blind mason to be solved at the cost of extended zone of GTP-tubulin depletion.

The final acts of tubulin docking are governed by the complexity of physical interactions. Firstly, due to the left-handed helical lattice symmetry of microtubule wall the docking tubulin interacts with tubulins, which already constitute the GTP-cap tip by means of spatially configured electrostatic interactions. Secondly, it also interacts with the other (competing) docking tubulins, and here are included electrostatic interactions, and excluded volume. Obviously tubulin, before its final addition to the GTP-cap tip, is undergoing strong nonlinear regime of cooperative interactions. Both, the strong interaction cooperativeness and nonlinearity phenomena are necessary ingredients in symmetry breaking during new structure formation [71]. In fact, single microtubule, as well as complex microtubules spatial patterns, manifests distinct symmetry features. On the other hand, from the thermodynamic point of view, since microtubule system is inherently open system, then so-called, spontaneous processes of microtubule growth and self-organization within the system are driven by the production of entropy, more specifically entropy decrease. Taking into account entropy decrease, breaking the symmetry, nonlinearity and cooperativeness of relevant physical interactions, and specific geometry of tubulin molecule, they all together endow the system as the whole with the power to resolve the enigma of blind mason by navigating the tubulin through adjusting its energy, position and proper orientation in order to be added to the GTP-cap tip.

In Figure 9, we have shown three possible classes of synergistic-topological patterns shaped by synergistic-topological mechanism. The first class (Figure 9A) is produced when the effects of local GTP-tubulin depletion and local cross diffusion are equilibrated. Dynamic instability occurs as a synergistic-topological function of those two. The corresponding biological example for this case may be seen in a polarized epithelial cell [57] (Figure 11A). The second class of fiber end-configuration topology is developed when the cross diffusion stabilization effects overwhelm the depletion effects (Figure 9B). This case is relevant for biological systems such as rod shaped cells of the fission yeast Schizosaccharomyces pombe [72] (see Figure 11B). The third class is generated when depletion effects overwhelm the local cross diffusion effects. This case is relevant for biological system of fibroblast, i.e. in formation of leading edge cortical polarity during fibroblast migration [72] (see Figure 11C).

Overall, intrinsic synergy mechanism in conjunction with topological features of the interface gap may control the gap size. Briefly, it was shown that when microtubules grow in a semi-confined volume, the parameters of microtubule dynamic instability and therefore the overall dynamic of microtubule growth, might be strongly influenced by subtle changes of the local GTP-tubulin concentration [30,73]. The multitude of neighboring microtubules imposes strong and uneven physical constraints on the GTP-tubulin influx into interface gap space. The impaired (tunable; single file) diffusion, i.e. cross diffusion as a particular kind of active diffusion is most likely the only mechanism by which GTP-tubulin may be brought, with a relative retardation, 
A

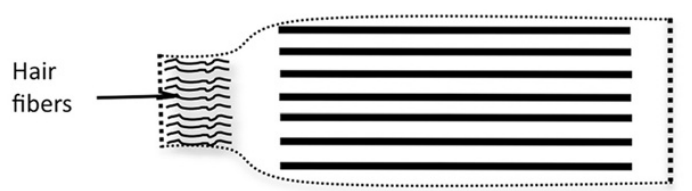

B

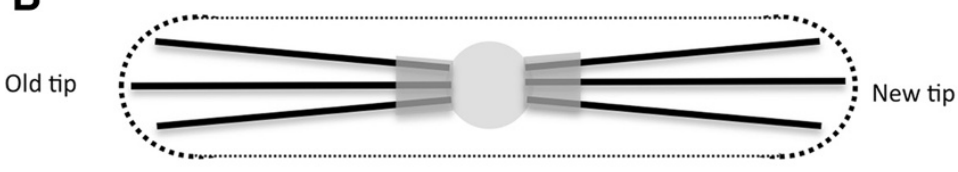

C

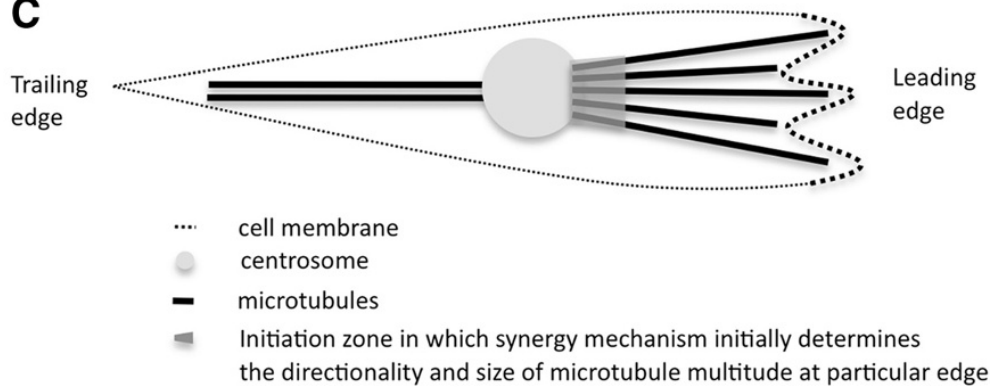

Figure 11 A biological examples of intrinsic control of size, shape and directionality of microtubule spatio-temporal pattern by synergistic-topological mechanism. A. Polarized epithelial cell (Schroer et al. [57]); local GTP-tubulin depletion and local cross diffusion are equilibrated. B. Microtubules intrinsic induction of cortical polarity in fission yeast (Schizosaccharomyces pombe) (Siegrist and Doe [72]); the cross diffusion stabilization effects overwhelm the depletion effects. C. Microtubules intrinsic induction of cortical polarity during fibroblast migration (Siegrist and Doe [72]); depletion effects overwhelm the local cross diffusion effects.

into a space of the interface gap [66,74-78]. Unevenly retarded GTP-tubulin cross-diffusion may influence internal local fluctuations of GTP-tubulin concentration within the gap. This local GTP-tubulin fluctuation may in conjunction with a topological stage of growing microtubule (within the gap) instigate a burst of GTP-hydrolysis, which in turn leads to dynamic instability. Consequently, the microtubule may start to shrink back and thus enlarge the interface gap. If the gap is enlarged to a certain size, the influx of fresh GTP-tubulin, by means of cross diffusion, may recommence and the microtubule growth may resume.

In conclusion, in the case of the interface gap, it is possible that tight correlation, i.e. synergy between dynamic instability, GTP-tubulin local fluctuations and cross

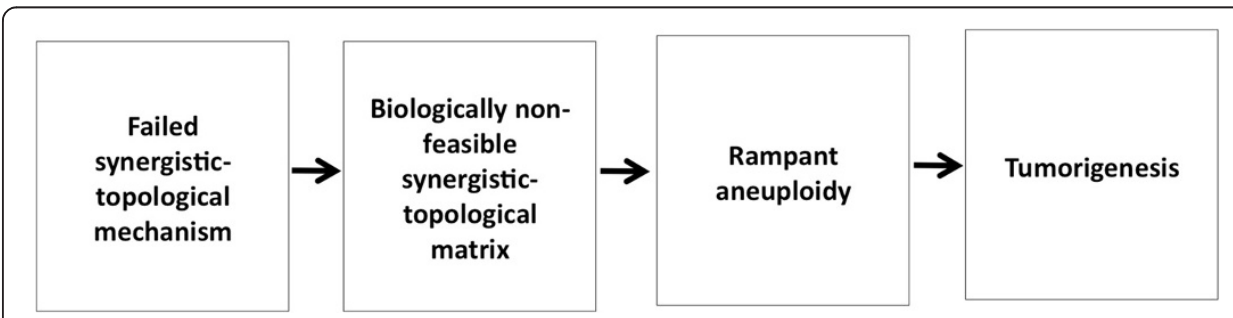

Figure 12 The possible role of microtubule synergistic-topological mechanism in tumorigenesis initiation. Failed synergistic-topological mechanism may initiate formation of biologically non-feasible microtubule synergistic-topological matrix. Non-feasible matrix mayenable rampant aneuploidy, that per se may preconditioned tumorigenesis to start (Duesberg, et al. [31]; Li, et al. [32]). 
(active) diffusion in conjunction with a topological stage of the interface gap microtubule controls the size of the gap. Thus, synergy mechanisms in conjunction with interface gap topology may be an important aspect of the controlling mechanism of force and energy transmission as well as signal processing along fiber microtubules $[26,39,58-61]$.

\section{Possible biological relevance of a synergistic-topological mechanism}

At biologically reduced conditions in vitro, we observed strong mutual interdependence of spatio-temporal microtubule pattern formation and locally associated solution conditions that may be explained by the existence of particular matrix. This matrix would be an integrated composite of microtubule spatio-temporal pattern and its specific locally associated solution conditions. The concept of a matrix related to the microtubule system of a mitotic spindle has already been proposed [23]. In our case, we consider that a synergistic-topological matrix is generated and maintained by the synergistic-topological mechanism including its modulator (the principle of summation of pairwise electrostatic forces between constituting microtubules of spatio-temporal microtubule pattern). If synergistic-topological mechanisms fail then it enables formation of biologically nonfeasible synergistic-topological matrices. This may further enable rampant aneuploidy that may per se precondition tumorigenesis [15,31,32] (Figure 12).

Competing interests

The authors declare that they have no competing interests.

\section{Authors contributions}

VAB conceptualized and designed the study, performed the experiments and wrote the manuscript; RMDH assisted with some of the experiments and assisted with the preparation of the manuscript; BDH critically evaluated the manuscript and assisted with the preparation of later versions; VK assisted with some of the experiments; EM assisted with some of the experiments; XL assisted with some of the experiments; GJL assisted with obtaining measurements described within the manuscript; JJB-F assisted with obtaining measurements described within the manuscript and RBB was involved in the discussions of the project concepts, evaluated the methodologies and assisted with the preparation of the manuscript. All authors read and approved the final manuscript.

\section{Acknowledgments}

We would like to express our sincere gratitude to Professor Maxwell Bennett, AO (Professor of Neuroscience, University Chair and Scientific Director, Brain and Mind Research Institute, The University of Sydney) for his genuine interest in this work and continued support.

We thank to microscopist Dr. Ian Kaplin (Australian Key Centre for Microscopy and Microanalysis, The University of Sydney) for technical assistance.

My personal (VB) sincere thanks to Mrs. Maria and Mr. Con for their kind help in the accomplishment of this work.

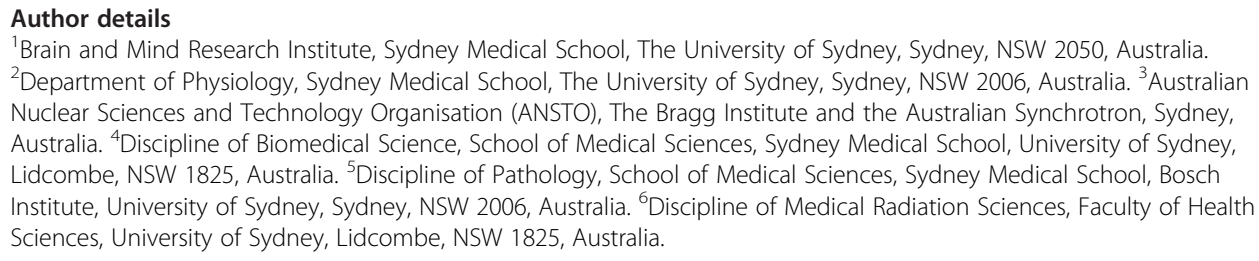

${ }^{1}$ Brain and Mind Research Institute, Sydney Medical School, The University of Sydney, Sydney, NSW 2050, Australia. ${ }^{2}$ Department of Physiology, Sydney Medical School, The University of Sydney, Sydney, NSW 2006, Australia. ${ }^{3}$ Australian Nuclear Sciences and Technology Organisation (ANSTO), The Bragg Institute and the Australian Synchrotron, Sydney, Australia. ${ }^{4}$ Discipline of Biomedical Science, School of Medical Sciences, Sydney Medical School, University of Sydney, Lidcombe, NSW 1825, Australia. ${ }^{5}$ Discipline of Pathology, School of Medical Sciences, Sydney Medical School, Bosch Institute, University of Sydney, Sydney, NSW 2006, Australia. ${ }^{6}$ Discipline of Medical Radiation Sciences, Faculty of Health Sciences, University of Sydney, Lidcombe, NSW 1825, Australia.

Received: 16 June 2014 Accepted: 31 October 2014

Published online: 04 December 2014

References

1. Neumann B, Hilliard MA: Loss of MEC-17 leads to microtubule instability and axonal degeneration. Cell Rep 2014, 6:93 103. doi:10.1016/J.Celrep.2013.12.004.

2. Etienne-Manneville S: Actin and microtubules in cell motility: which one is in control? Traffic 2004, 5:470 477. doi:10.1111/J.1600-0854.2004.00196.X.

3. Tamura N, Draviam VM: Microtubule plus-ends within a mitotic cell are moving platforms with anchoring, signalling and force-coupling roles. Open Biol 2012, 2:Artn 120132. doi:10.1098/Rsob.120132.

4. Gerlitz G, Reiner O, Bustin M: Microtubule dynamics alter the interphase nucleus. Cell Mol Life Sci 2013, 70:1255 1268. doi:10.1007/S00018-012-1200-5. 
5. Mitsuyama F, Kato K, Hirosawa K, Mikoshiba K, Okuya M, Karagiozov K, Kato Y, Kanno T, Sanoe H, Koide T: Redistribution of microtubules in dendrites of hippocampal CA1 neurons after tetanic stimulation during long-term potentiation. Ital J Anat Embryol 2008, 17:27.

6. Craddock TJ, Tuszynski JA, Hameroff S: Cytoskeletal signaling: is memory encoded in microtubule lattices by CaMKII phosphorylation? PLoS Comput Biol 2012, 8:e1002421. doi:10.1371/journal.pcbi.1002421.

7. Akhshi TK, Wernike D, Piekny A: Microtubules and actin crosstalk in cell migration and division. Cytoskeleton 2014, 71:1 23. doi:10.1002/Cm.21150.

8. Bouissou A, Verollet C, de Forges H, Haren L, Bellaiche Y, Perez F, Merdes AB: Raynaud-Messina: gamma-Tubulin ring complexes and EB1 play antagonistic roles in microtubule dynamics and spindle positioning. Embo J 2014, 33:114 128. doi:10.1002/Embj.201385967.

9. Zanic M, Widlund PO, Hyman AA, Howard J: Synergy between XMAP215 and EB1 increases microtubule growth rates to physiological levels. Nat Cell Biol 2013, 15:688. doi:10.1038/Ncb2744.

10. Janke C, Kneussel M: Tubulin post-translational modifications: encoding functions on the neuronal microtubule cytoskeleton. Trends Neurosci 2010, 33:362 372. doi:10.1016/J.Tins.2010.05.001.

11. Stoppin-Mellet V, Fache V, Portran D, Martiel JL, Vantard M: MAP65 coordinate microtubule growth during bundle formation. Plos One 2013, 8:ARTN e56808. doi:10.1371/journal.pone.0056808.

12. Mitchison T, Whr M, Mitchison P, Nguyen K, Ishihara A, Groen CM: Field: growth, interaction and positioning of microtubule asters in extremely large vertebrate embryo cells. Cytoskeleton (Hoboken) 2012, 69:738 750

13. Wuhr M, Dumont $\mathrm{S}$, Groen $A C$, Needleman DJ, Mitchison TJ: How does a millimeter-sized cell find its center? Cell Cycle 2009, 8:1115 1121.

14. Smurova AABKM, Verin AD, Alieva IB: Microtubule system in endothelial barrier dysfunction: disassembly of peripheral microtubules and microtubule reorganization in internal cytoplasm. Cell Tissue Biol 2008, 2:45 52

15. Sayas CJ, Avila J: Crosstalk between axonal classical microtubule-associated proteins and end binding proteins during axon extension: possible implications in neurodegeneration. J Alzheimers Dis 2013, x:xx.

16. Gavin RH: Synergy of cytoskeleton components - cytoskeletal polymers exhibit both structural and functional synergy. Bioscience 1999, 49:641 655. doi:10.2307/1313440.

17. Johansen KM, Johansen J: Recent glimpses of the elusive spindle matrix. Cell Cycle 2002, 1:312 314

18. Teng J, Takei Y, Harada A, Nakata T, Hirokawa N: Synergistic effects of MAP2 and MAP1B knockout in neuronal migration, dendritic outgrowth, and microtubule organization. Mol Biol Cell 2001, 12:433A.

19. Clausen T, Ribbeck K: Self-organization of anastral spindles by synergy of dynamic instability, autocatalytic microtubule production, and a spatial signaling gradient. Plos One 2007, 2:ARTN e244 doi:10.1371/journal.pone.0000244.

20. Schuh M, Ellenberg J: Self-organization of MTOCs replaces centrosome function during acentrosomal spindle assembly in live mouse oocytes. Cell 2007, 130:484 498. doi:10.1016/J.Cell.2007.06.025.

21. Karsenti E, Vernos I: Cell cycle - the mitotic spindle: a self-made machine. Science 2001, 294:543 547. doi:10.1126/Science.1063488.

22. Brunet S, Polanski Z, Verlhac MH, Kubiak JZ, Maro B: Bipolar meiotic spindle formation without chromatin. Curr Biol 1998, 8:1231 1234. doi:10.1016/S0960-9822(07)00516-7.

23. Pickettheaps JD, Tippit DH, Porter KR: Rethinking Mitosis. Cell 1982, $29: 729$ 744. doi:10.1016/0092-8674(82)90435-4.

24. Lince-Faria M, Maffini S, Orr B, Ding Y, Florindo C, Sunkel CE, Tavares A, Johansen J, Johansen KM, Maiato H: Spatiotemporal control of mitosis by the conserved spindle matrix protein Megator. J Cell Biol 2009, 184:647 657. doi:10.1083/Jcb.200811012.

25. Johansen KM, Johansen J: Cell and molecular biology of the spindle matrix. Int Rev Cytol 2007, 263:155 doi10.1016/S0074-7696(07)63004-6.

26. Johansen KM, Forer A, Yao CF, Girton J, Johansen J: Do nuclear envelope and intranuclear proteins reorganize during mitosis to form an elastic, hydrogel-like spindle matrix? (vol 19, pg 345, 2011). Chromosome Res 2011, 19:683. doi:10.1007/S10577-011-9217-4.

27. Buljan VA, Holsinger RMD, Brown D, Bohorquez-Florez JJ, Hambly BD, Delikatny EJ, Ivanova EP, Banati RB: Spinodal decomposition and the emergence of dissipative transient periodic spatio-temporal patterns in acentrosomal microtubule multitudes of different morphology. Chaos 2013, 23:Artn 023120. doi10.1063/1.4807909

28. Dlugosz $\mathrm{M}$, Antosiewicz JM: Evaluation of proteins rotational diffusion coefficients from simulations of their free brownian motion in volume-occupied environments. J Chem Theory Comput 2014, 10:481 491. doi:10.1021/Ct4008519.

29. Hunyadi V, Chretien D, Flyvbjerg H, Janosi IM: Why is the microtubule lattice helical? Biol Cell 2007, 99:117 128. doi:10.1042/Bc20060059.

30. Podgornik R, Parsegian VA: Charge-fluctuation forces between rodlike polyelectrolytes: pairwise summability reexamined. Phys Rev Lett 1998, 80:1560 1563. Doi: 10.1103/Physrevlett.80.1560.

31. Duesberg P, Li RH, Fabarius A, Hehlmann R: The chromosomal basis of cancer. Cell Oncol 2005, 27:293 318

32. Li RH, Yerganian G, Duesberg P, Kraemer A, Willer A, Rausch C, Hehlmann R: Aneuploidy correlated 100\% with chemical transformation of Chinese hamster cells. P Natl Acad Sci USA 1997, 94:14506 14511. doi:10.1073/Pnas.94.26.14506.

33. Shelansk M, Gaskin F, Cantor CR: Microtubule assembly in absence of added nucleotides. P Natl Acad Sci USA 1973, 70:765 768. doi10.1073/Pnas.70.3.765.

34. Langford GM: Length and appearance of projections on neuronal microtubules invitro after negative staining - evidence against a crosslinking function for maps. J Ultra Mol Struct R 1983, 85:1 10. doi:10.1016/S0022-5320(83)90111-9.

35. Buljan V, Ivanova EP, Cullen KM: How calcium controls microtubule anisotropic phase formation in the presence of microtubule-associated proteins in vitro. Biochem Bioph Res Co 2009, 381:224 228.

36. Turner DC, Chang CY, Fang K, Brandow SL, Murphy DB: Selective adhesion of functional microtubules to patterned silane surfaces. Biophys J 1995, 69:2782 2789. 
37. Anderson PW: More is different - broken symmetry and nature of hierarchical structure of science. Science 1972, 177:393. doi:10.1126/Science.177.4047.393.

38. Corning PA: Synergy and self-organization in the evolution of complex-systems. Syst Res 1995, 12:89 121. doi:10.1002/Sres.3850120204.

39. Tuszynski JA, Hameroff S, Sataric MV, Trpisova B, Nip MLA: Ferroelectric behavior in microtubule dipole lattices - implications for information-processing: signaling and assembly disassembly. J Theor Biol 1995, 174:371 380 .

40. Tuszynski JA, Malinski W, Carpenter EJ, Luchko T, Huzil JT, Ludena RF: Tubulin electrostatics and isotype specific drug binding. Can J Phys 2008, 86:635 640. doi:10.1139/P07-199.

41. Barton JS, Vandivort DL, Heacock DH, Coffman JA, Trygg KA: Microtubule assembly kinetics - changes with solution conditions. Biochem J 1987, 247:505 511.

42. Tuszynski JA, Brown JA, Crawford E, Carpenter EJ, Nip MLA, Dixon JM, Sataric MV: Molecular dynamics simulations of tubulin structure and calculations of electrostatic properties of microtubules. Math Comput Model 2005, 41:1055 1070. doi:10.1016/J.Mcm.2005.05.002.

43. Zimmerman SB, Minton AP: Macromolecular crowding - biochemical, biophysical, and physiological consequences. Annu Rev Bioph Biom 1993, 22:27 65. doi:10.1146/Annurev.Bb.22.060193.000331.

44. Odde DJ: Estimation of the diffusion-limited rate of microtubule assembly. Biophys J 1997, 73:88 96.

45. Shelden E, Wadsworth P: Observation and quantification of individual microtubule behavior invivo microtubule dynamics are cell-type specific. J Cell Biol 1993, 120:935 945. doi:10.1083/Jcb.120.4.935.

46. Tabony J: Morphological bifurcations involving reaction diffusion processes during microtubule formation. Science 1994, 264:245 248. doi:10.1126/Science.8146654.

47. Hall D, Minton AP: Macromolecular crowding: qualitative and semiquantitative successes, quantitative challenges. Bba-Proteins Proteom 2003, 1649:127 139. doi:10.1016/S1570-9639(03)00167-5.

48. Minton AP: The influence of macromolecular crowding and macromolecular confinement on biochemical reactions in physiological media. J Biol Chem 2001, 276:10577 10580.

49. Minton AP: Effects of excluded surface area and adsorbate clustering on surface adsorption of proteins: II. Kinetic models. Biophys J 2001, 80:1641 1648.

50. Matthews BW, Remingto SJ: 3 dimensional structure of lysozyme from bacteriophage-T4. P Natl Acad Sci USA 1974, 71:4178 4182. doi:10.1073/Pnas.71.10.4178.

51. Nogales E, Whittaker M, Milligan RA, Downing KH: High-resolution model of the microtubule. Cell 1999, 96:79 88. doi:10.1016/S0092-8674(00)80961-7.

52. Herzfeld J: Crowding-induced organization in cells: spontaneous alignment and sorting of filaments with physiological control points. J Mol Recognit 2004, 17:376 381.

53. Onsager L: The effects of shape on the interaction of colloidal particles. Ann Ny Acad Sci 1949, 51:627 659. doi:10.1111/J.1749-6632.1949.Tb27296.X.

54. Liu YF, Guo YX, Valles JM, Tang JX: Microtubule bundling and nested buckling drive stripe formation in polymerizing tubulin solutions. P Natl Acad Sci USA 2006, 103:10654 10659. doi:10.1073/Pnas.0510381103.

55. Hitt AL, Cross AR, Williams RC: Microtubule solutions display nematic liquid-crystalline structure. J Biol Chem 1990, 265:1639 1647.

56. Baas PW, Ahmad FJ: Beyond taxol: microtubule-based treatment of disease and injury of the nervous system. Brain 2013, 136:2937 2951. doi:10.1093/Brain/Awt153.

57. Schroer TA, Sheetz MP: Functions of Microtubule-Based Motors. Annu Rev Physiol 1991, 53:629 652. doi:10.1146/Annurev.Physiol.53.1.629.

58. Sekulic DL, Sataric BM, Tuszynski JA, Sataric MV: Nonlinear ionic pulses along microtubules. Eur Phys J E 2011, 34:Artn 49. doi:10.1140/Epje//2011-11049-0.

59. Das M, Levine AJ, MacKintosh FC: Buckling and force propagation along intracellular microtubules. Epl-Europhys Lett 2008, 84:Artn 18003. doi:10.1209/0295-5075/84/18003.

60. Sataric MV, Tuszynski JA, Zakula RB: Kink-like excitations as an energy-transfer mechanism in microtubules. Phys Rev E 1993, 48:589 597. doi:10.1103/Physreve.48.589.

61. Hameroff SR, Watt RC: Information-processing in microtubules. J Theor Biol 1982, $98: 549561$.

62. Rodriguez OC, Schaefer AW, Mandato CA, Forscher P, Bement WM, Waterman-Storer CM: Conserved microtubule-actin interactions in cell movement and morphogenesis. Nat Cell Biol 2003, 5:599 609. doi:10.1038/Ncb0703-599.

63. Kirschner M, Mitchison T: Beyond self-assembly - from microtubules to morphogenesis. Cell 1986, 45:329 342.

64. Tabony J, Vuillard L, Papaseit C: Biological self-organisation and pattern formation by way of microtubule reaction diffusion processes. Adv Complex Syst 1999, 02:221 276. doi:10.1142/S0219525999000138.

65. Buljan VA, Holsinger RMD, Hambly BD, Banati RB, Ivanova EP: Intrinsic microtubule GTP-cap dynamics in semi-confined systems: kinetochore-microtubule interface. J Biol Phys 2013, 39:81 98. doi:10.1007/S10867-012-9287-3.

66. Brangwynne CP, Koenderink GH, MacKintosh FC, Weitz DA: Intracellular transport by active diffusion. Trends Cell Biol 2009, 19:423 427. doi:10.1016/J.Tcb.2009.04.004.

67. Brangwynne CP, Koenderink GH, MacKintosh FC, Weitz DA: Nonequilibrium microtubule fluctuations in a model cytoskeleton. Phys Rev Lett 2008, 100:Artn 118104. doi10.1103/Physrevlett.100.118104.

68. Chasey D: Left-handed subunit helix in flagellar microtubules. Nature 1974, 248:611 612. doi:10.1038/248611a0.

69. Amos LA, Klug A: Arrangement of subunits in flagellar microtubules. J Cell Sci 1974, 14:523 549.

70. Mandelkow EM, Schultheiss R, Rapp R, Muller M, Mandelkow E: On the surface lattice of microtubules - helix starts, protofilament number, seam, and handedness. J Cell Biol 1986, 102:1067 1073. doi:10.1083/Jcb.102.3.1067.

71. Ebeling W: Strukturbildung bei irreversiblen prozessen. Leipzig: B.G. Teubner Verlagsgesellschaft; 1976.

72. Siegrist SE, Doe CQ: Microtubule-induced cortical cell polarity. Gene Dev 2007, 21:483 496. doi:10.1 101/Gad.1511207.

73. Janulevicius A, van Pelt J, van Ooyen A: Compartment volume influences microtubule dynamic instability: a model study. Biophys J 2006, 90:788 798. Doi: 10.1529/Biophysj.105.059410. 